\title{
Elliptic symbols, elliptic operators and Poincaré duality on conical pseudomanifolds
}

\author{
Jean-Marie Lescure
}

July 25, 2018

\begin{abstract}
In 17, a notion of noncommutative tangent space is associated with a conical pseudomanifold and the Poincare duality in $K$-theory is proved between this space and the pseudomanifold. The present paper continues this work. We show that an appropriate presentation of the notion of symbols on a manifold generalizes right away to conical pseudomanifolds and that it enables us to interpret the Poincaré duality in the singular setting as a noncommutative symbol map.
\end{abstract}

\section{Contents}

1 Introduction 1

2 Equivalences of tangent spaces and Dirac elements 7

3 Cycles of the $K$-theory of the tangent space 10

4 Poincaré dual of elliptic noncommutative symbols 18

\section{Introduction}

In this paper we give a concrete description of the Poincaré duality in $K$-theory for a conical pseudomanifold as stated and proved in [7. This duality holds between the algebra $C(X)$ of continuous fonctions on a (compact) pseudomanifold $X$ and the $C^{*}$-algebra $C^{*}\left(T^{c} X\right)$ of a suitable tangent space of this pseudomanifold.

The tangent space $T^{c} X$ introduced in [7] is a smooth groupoid. It is no more commutative, but it restricts to the usual tangent space of a manifold outside the singularity and the singular contribution is quite simple.

The duality between $C(X)$ and $C^{*}\left(T^{c} X\right)$ is defined in terms of bivariant $K$-theory but it is important to recall that it implies the existence of an isomorphism:

$$
\Sigma^{c}: K_{0}(X) \stackrel{\simeq}{\longrightarrow} K_{0}\left(C^{*}\left(T^{c} X\right)\right)
$$

The main purpose of this paper is to identify this isomorphism with a noncommutative symbol map, as one does in the smooth case with the usual symbol map. Indeed, the Poincaré duality in the case of a smooth closed manifold $V$ induces an isomorphism between $K_{0}(V)$ and $K_{0}\left(C^{*}(T V)\right) \simeq K^{0}\left(T^{*} V\right)$ which is nothing else but the principal symbol map:

$$
\begin{aligned}
& K_{0}(V) \longrightarrow K^{0}\left(T^{*} V\right) \\
& {[P] \quad \longmapsto \quad[\sigma(P)]}
\end{aligned}
$$

sending classes of elliptic pseudodifferential operators (the basic cycles of the $K$-homology of $V$ ) to classes of their principal symbols (the basic cycles of the $K$-theory with compact supports of $\left.T^{*} V\right)$. 
The interpretation of (1.1) as a noncommutative symbol map is really important for two reasons. Firstly, it validates the choice of a tangent space which is $K$-dual to the singular manifold and thus motivates further investigations toward analysis or differential geometry of singular spaces by using this noncommutative object as well as other tools of noncommutative geometry. Secondly, this approach can be of interest for people looking for Fredholmness conditions in elliptic equations in singular situations like stratified spaces. Indeed, the notion of tangent space of a stratified space is very intuitive as soon as one understands the conical case, and the notion of elliptic noncommutative symbols appears directly. The case of general stratifications will be treated in forthcoming articles.

In [7, we propose two $K K$-equivalent definitions of the tangent space of a pseudomanifold $X$ and the main results were stated for the first one, noted $T^{c} X$ in the present article. To explain in what sense cycles of the $K$-theory of the tangent space of a pseudomanifold are noncommutative symbols and cycles of its $K$-homology are pseudodifferential operators, we will use here the second definition given in [7], noted in the sequel $T^{q} X$. The equivalence in $K$-theory of both tangent spaces allows us to state all the results of [7] for $T^{q} X$ and in particular the isomorphism (1.1). Even if this equivalence is obvious for people familiar with groupoids, one will give full details about it in section 2 .

Now, surprisingly, one can define noncommutative symbols on a pseudomanifold exactly as one defines symbols on a smooth manifold. More precisely, symbols on a smooth manifold $V$ are functions on the cotangent space $T^{*} V$ with adequate behavior in the fibers. They can be considered as pointwise multiplication operators on, for instance, $C_{c}^{\infty}\left(T^{*} V\right)$. Under a Fourier transform in the fibers, they can also be viewed as families parametrized by $V$ of convolutions operators in the fibers of $T V$. Thus:

Symbols on $V$ are pseudodifferential operators on the tangent space $T V$, where $T V$ is considered as a groupoid and we talk about pseudodifferential calculus for groupoids [21, 18, 28, 27].

This simple observation is already important to understand that the tangent groupoid defined by A. Connes in [5] gives the analytic index of elliptic pseudodifferential operators. Next, it suggests the following definition of noncommutative symbols on the pseudomanifold $X$.

Noncommutative symbols on $X$ are pseudodifferential operators on the tangent space $T^{q} X$.

We will see that, after some technical precautions on the Schwartz kernels and on the behavior near the "end" of $T^{q} X$ of these pseudodifferential operators, this apparently naive idea works. For instance, one can recover in a single object the notions of interior and conormal symbols arising in boundary problems and the notion of full ellipticity is quite immediate here.

Concerning the operators involved in the description of the Poincaré duality, some freedom is allowed: basically, all calculi based on the work of R. Melrose [14] ( $b$ or $c$ calculi for instance) as well as on the work of B.W. Schulze [23, 24] can be used indifferently and lead to various representants of the same $K$-homology class (that is, to the Poincaré dual of a given elliptic noncommutative symbol).

The main tools used in this paper are Lie groupoids (see [7] and the corresponding bibliography), pseudodifferential calculus (see [25, 14, 18, 21, 27]) bivariant $K$-theory (see [11, 12, 26, 6, 3, 29, 2]).

The author mentions that different techniques have been precedently used to produce results close from the present work by A. Savin ([22], see also joint works by V. Nazaikinskii, A. Savin and B. Sternin [20, 19]).

\section{$1.1 \quad$ Reviews and Notations}

The range and source maps of groupoids are noted $r$ and $s$. If $A$ is a subset of the space of units $G^{(0)}$ of a groupoid $G$ then $\left.G\right|_{A}$ denotes the subgroupoid $\left.G\right|_{A}=r^{-1}(A) \cap s^{-1}(A)$. 
All groupoids in the sequel are smooth (Lie groupoids), endowed with Haar systems in order to define their $C^{*}$-algebras. Moreover, they are amenable (as continuous fields of amenable groupoids [1]). In particular, there is no ambiguity about their $C^{*}$-algebras and notations for their $K$-theory will be shortened:

$$
K^{i}(G):=K_{i}\left(C^{*}(G)\right) \text { and } K K\left(G_{1}, G_{2}\right):=K K\left(C^{*}\left(G_{1}\right), C^{*}\left(G_{2}\right)\right)
$$

If $f$ is a homomorphism between two $C^{*}$-algebras $A, B$, the corresponding class in $K K(A, B)$ will be denoted by $[f]$.

When a vector bundle $E \rightarrow G^{(0)}$ is given, we define a $C^{*}(G)$-Hilbert module noted $C^{*}(G, E)$ by taking the completion of $C_{c}^{\infty}\left(G, r^{*} E\right)$ for the norm associated with the $C^{*}(G)$ valued product :

$$
<f, g>(\gamma)=\int_{\eta \in G^{r(\gamma)}}<f\left(\eta^{-1}\right), g\left(\eta^{-1} \gamma\right)>_{s(\eta)}
$$

We shall use various deformation groupoids $\left.\left.G=G_{1} \times\{t=0\} \cup G_{2} \times\right] 0,1\right]_{t}$. The restriction morphism $\mathrm{ev}_{t=0}: G \rightarrow G_{1}$ at $t=0$ gives an exact sequence:

$$
\left.\left.0 \rightarrow C^{*}\left(G_{2} \times\right] 0,1\right]\right) \rightarrow C^{*}(G) \stackrel{\mathrm{ev}_{t=0}}{\rightarrow} C^{*}\left(G_{1}\right) \rightarrow 0
$$

whose ideal is contractible in $K K$-theory. If $G_{1}$ is amenable (which will always be the case in this paper), one gets that $\left[\operatorname{ev}_{t=0}\right] \in K K\left(G, G_{2}\right)$ is invertible. The deformation element associated with the deformation groupoid is the Kasparov element defined by

$$
\partial_{G}=\left[\mathrm{ev}_{t=0}\right]^{-1} \otimes\left[\mathrm{ev}_{t=1}\right] \in K K\left(G_{1}, G_{2}\right)
$$

For convenience, the pair groupoid on a set $E$ will be denoted by $\mathcal{C}_{E}$.

The (open) cone over a space $L$ is the quotient space $c L=(L \times[0,+\infty[) / L \times\{0\}$.A conical pseudomanifold is a compact metrisable space $X$ equipped with the following data. There is one singular point (but everything in the sequel can be written for a finite number) which means that a point $c \in X$ is given and that $X^{o}:=X \backslash\{c\}$ is a manifold. Moreover there is an open neighborhood $\mathcal{N}$ of $c$, a smooth manifold $L$, continuous maps $h: \mathcal{N} \rightarrow\left[0,+\infty\left[\right.\right.$ and $\varphi_{c}: \mathcal{N} \rightarrow c L$ satisfying the following:

- $h$ is surjective, $h^{-1}\{0\}=\{c\}$ and $\left.h: \mathcal{N} \backslash\{c\} \rightarrow\right] 0,+\infty[$ is a smooth submersion,

- $\varphi_{c}: \mathcal{N} \rightarrow c L$ is a homeomorphism, smooth outside $c$, such that:

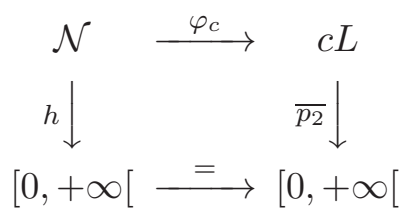

commutes. Here $\overline{p_{2}}$ denotes the quotient map of the second projection $L \times \mathbb{R}_{+} \rightarrow \mathbb{R}_{+}$.

Conical pseudomanifolds are the simplest examples of a stratified space [4]. We distinguish two parts in the regular stratum $X^{o}$ :

$$
X^{o}=X_{-} \cup X_{+}
$$

where $\left.X_{-}=h^{-1}\right] 0,1\left[\right.$, and $X_{+}=X \backslash X_{-}$is a smooth compact manifold with boundary, the latter being identified with $L$. The identification $\left.X_{-} \simeq\right] 0,1\left[\times L\right.$ given by $\varphi_{c}$ will be often used without mention. The compactification $M=\overline{X^{o}}$ of $X^{o}$ into a manifold with boundary $L$ will be sometimes useful. The following picture illustrates the notations just defined: 

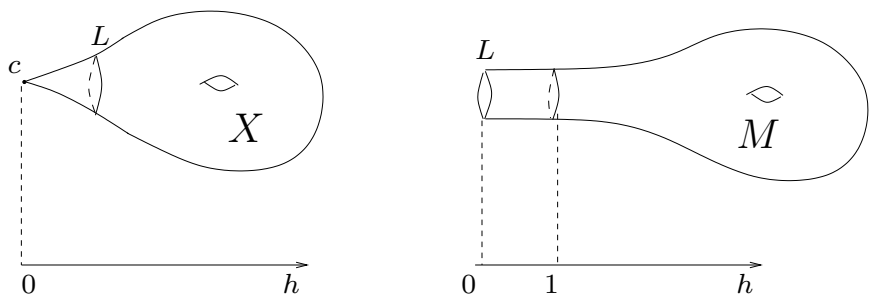

$$
\begin{aligned}
& h(X \backslash \mathcal{N}):=+\infty \\
& \left.\left.X^{o}=h^{-1}(] 0,+\infty\right]\right) \\
& X_{+}=h^{-1}([1,+\infty]) \\
& \left.X_{-}=h^{-1}\right] 0,1[ \\
& M=h^{-1}([0,+\infty])
\end{aligned}
$$

A riemannian metric $g$ on $X^{o}$ satisfying:

$$
\left.\left(\varphi_{c}\right)_{*} g(h, y)=d h^{2}+g_{L}(y), \quad(h, y) \in\right] 0,+\infty[\times L
$$

on $\mathcal{N} \backslash\{c\}$ is chosen, where $g_{L}$ is a riemannian metric on $L$. The corresponding exponential maps for $X^{o}$ and $L$ are denoted $e$ and $e_{L}$, and the injectivity radius is assumed to be greater than 1 in both cases. The geodesic distances are denoted dist, dist $L_{L}$. The associated riemannian measure on $X^{o}$ and $L$ will be noted $d \mu$ and $d \mu_{L}$ and the associated Lebesgue measures on $T_{x} X^{o}$ and $T_{x}^{*} X^{o}$ for $x \in X^{o}$ will be noted $d X$ and $d \xi$. We will note $d \mu^{\mathbb{R}}$ the Lebesgue measure on $\mathbb{R}$.

We shall assume that $X^{o}$ is oriented, but all constructions below can be done in the general case with half densities bundles.

The tangent space of a conical pseudomanifold $X$ was defined in [7] by:

$$
T^{c} X=\mathcal{C}_{X_{-}} \cup T X_{+} \rightrightarrows X^{o}
$$

The unit space is $X^{o}$. This is a disjoint union where $\mathcal{C}_{X_{-}}$is the pair groupoid of $X_{-}$and $T X_{+}$has groupoid structure equal to its vector bundle structure. We will mainly use in this paper a slightly different but equivalent (at the level of $K$-theory) definition of the tangent space which was also given in [7]:

$$
\left.T^{q} X=T\right] 0,1\left[{ }_{h} \times \mathcal{C}_{L} \cup T X_{+} \rightrightarrows X^{o}\right.
$$

We will refer to (1.7) as the 'q' version and (1.6) as the 'c' version of the tangent space of $X$.

The tangent groupoid is defined for the 'c' and the 'q' version by:

$$
\begin{aligned}
\mathcal{G}^{c} & \left.\left.=T^{c} X \times\{0\} \cup \mathcal{C}_{X^{o}} \times\right] 0,1\right]_{t} . \\
\mathcal{G}^{q} & \left.\left.=T^{q} X \times\{0\} \cup \mathcal{C}_{X^{o}} \times\right] 0,1\right]_{t}
\end{aligned}
$$

In order to write down on $T^{q} X$ some constructions made in [7] for $T^{c} X$, one needs the following deformation groupoids:

$$
\begin{gathered}
\left.\left.H=T^{q} X \times\{u=0\} \cup T^{c} X \times\right] 0,1\right]_{u}, \\
\left.\left.\mathcal{H}=\mathcal{G}^{q} \times\{u=0\} \cup \mathcal{G}^{c} \times\right] 0,1\right]_{u}
\end{gathered}
$$

Let us recall that $\mathcal{H}$ has three deformation parameters noted $h, t, u$ and contains all previous groupoids:

$$
\left.\mathcal{H}\right|_{t=0}=H,\left.\mathcal{H}\right|_{u=0}=\mathcal{G}^{q},\left.\mathcal{H}\right|_{u=1}=\mathcal{G}^{c},\left.\mathcal{H}\right|_{t=0, u=0}=T^{q} X,\left.\mathcal{H}\right|_{t=0, u=1}=T^{c} X .
$$




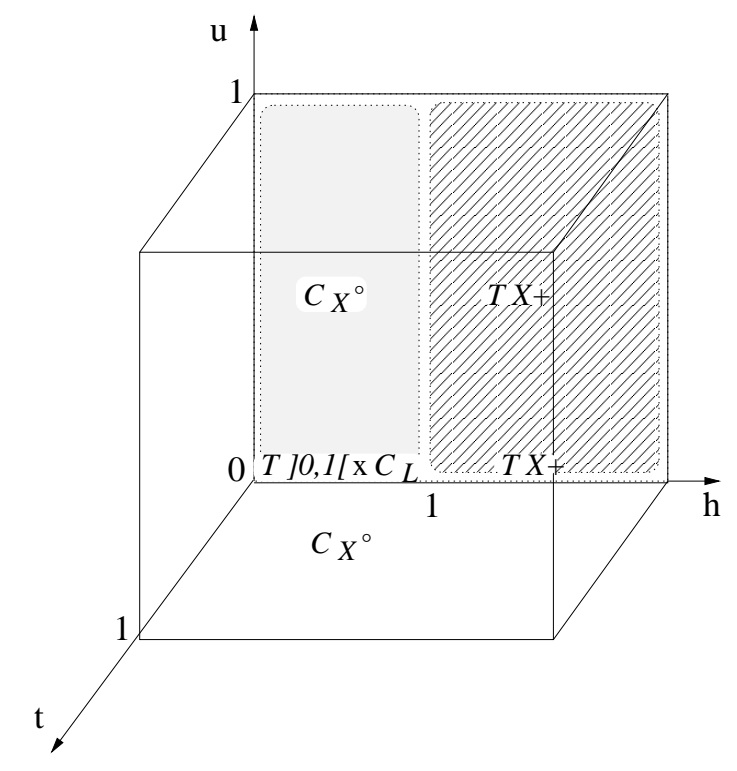

We describe now a differentiable structure for $\mathcal{H}$. This is rather technical, and the unfamiliar reader can skip this construction up to the remark 1 which will be reused later.

The unit space of $\mathcal{H}$ is $\mathcal{H}^{0}=X^{o} \times[0,1]_{t, u}^{2}$. We cover $\mathcal{H}^{0}$ by four open subsets : $\mathcal{H}^{0}=A \cup B \cup C \cup D$ with:

$$
\begin{array}{cc}
A=\operatorname{int}\left(X_{+}\right) \times[0,1]_{t, u}^{2} & \left.\left.B=X^{o} \times\right] 0,1\right]_{t} \times[0,1]_{u} \\
C=X_{-} \times[0,1]_{t, u}^{2} & D=h^{-1}(] 1-\varepsilon, 1+\varepsilon[) \times\left[0, \varepsilon\left[{ }_{t} \times[0,1]_{u}\right.\right.
\end{array}
$$

Here $\varepsilon$ is an arbitrary small number and $\operatorname{int}\left(X_{+}\right)=X_{+} \backslash \partial X_{+}$. We get the following cover of $\mathcal{H}$ :

$$
\mathcal{H}=\left.\left.\left.\left.\mathcal{H}\right|_{A} \cup \mathcal{H}\right|_{B} \cup \mathcal{H}\right|_{C} \cup \mathcal{H}\right|_{D}
$$

We have $\left.\mathcal{H}\right|_{A}=\mathcal{G}_{\operatorname{int}\left(X_{+}\right)} \times[0,1]_{u}$ where $\mathcal{G}_{\operatorname{int}\left(X_{+}\right)}$is the tangent groupoid [5] of $\operatorname{int}\left(X_{+}\right)$. We provide it with its usual smooth structure and $\left.\mathcal{H}\right|_{A}$ inherits the obvious product smooth structure. Next, the smooth structure of $\left.\left.\left.\mathcal{H}\right|_{B}=\mathcal{C}_{X^{o}} \times\right] 0,1\right]_{t} \times[0,1]_{u}$ is the product one. For the two remaining subgroupoids, we need to specify some gluing functions in the deformation parameters. We choose once for all a smooth decreasing function:

$$
\tau:[0,+\infty[\rightarrow[0,1]
$$

satisfying $\tau(h)=1$ on $[0,1 / 2]$ and $\tau^{-1}\{0\}=[1,+\infty[$. Let:

$$
\kappa:[0,+\infty[\times[0,1] \rightarrow[0,1]
$$

be a smooth function satisfying $\min (1, \tau(h)+t) \leqslant \kappa(h, t) \leqslant 1$ and $\kappa(h, t)=\tau(h)+t$ if $\tau(h)+t \leqslant 3 / 4$. Let:

$$
\mu:[0,+\infty[\times[0,1] \times[0,1] \rightarrow[0,1]
$$

be a smooth function satisfying $\min (1, u \tau(h)+t) \leqslant \mu(h, t, u) \leqslant 1$ and $\mu(h, t, u)=u \tau(h)+t$ if $u \tau(h)+t \leqslant 3 / 4$. Let:

$$
\left.l: \mathbb{R}_{+}=\right] 0,+\infty[\rightarrow \mathbb{R}
$$

be a smooth bijective function satisfying $\frac{d}{d h} l>0$ and $l=$ Id on a neighborhood of $[1,+\infty[$ in $] 0,+\infty[$.

Coming back to the subgroupoid $\left.\mathcal{H}\right|_{C}$, observe that:

$$
\left.\mathcal{H}\right|_{C}=(T] 0,1\left[{ }_{h} \times\{(0,0)\} \cup \mathcal{C}_{0,1\left[\left[_{h}\right.\right.} \times[0,1]_{t, u}^{2} \backslash\{(0,0)\}\right) \times \mathcal{C}_{L}
$$


We note shortly $G_{I}$ the first factor in $\left.\mathcal{H}\right|_{C}$. Let $\mathcal{V}$ be the open subset of $\left.T\right] 0,1\left[{ }_{h} \times[0,1]_{t, u}^{2}\right.$ given by:

$$
\mathcal{V}=\left\{(h, \lambda, t, u) \mid l^{-1}(l(h)+\mu(h, t, u) \lambda)<1\right\}
$$

Then we provide $G_{I}$ with the smooth structure such that the bijective map $E_{G_{I}}: \mathcal{V} \rightarrow G_{I}$ given by:

$$
E_{G_{I}}(h, \lambda, t, u)=\left\{\begin{array}{cc}
(h, \lambda, 0,0) & \text { if }(t, u)=(0,0) \\
\left(h, l^{-1}(l(h)+\mu(h, t, u) \lambda), t, u\right) & \text { if }(t, u) \neq(0,0)
\end{array}\right.
$$

is a diffeomorphism. Thus, $\left.\mathcal{H}\right|_{C}$ inherits the product structure of $G_{I} \times \mathcal{C}_{L}$.

It is tedious but not difficult to check that the smooth structures given to $\left.\mathcal{H}\right|_{A},\left.\mathcal{H}\right|_{B},\left.\mathcal{H}\right|_{C}$ are compatible on their common domain and it remains to give $\left.\mathcal{H}\right|_{D}$ with a compatible smooth structure.

Remember that $X^{o}$ and $L$ are riemmannian with exponential maps denoted by $e$ and $e^{L}$ (see paragraph 1.1) and consider now the open subset $\mathcal{U}$ of $T X^{o} \times[0,1]_{t, u}^{2}$ given by the set of $(x, V, t, u) \in T X^{o} \times[0,1]^{2}$ satisfying:

- if $h(x) \geqslant 1$ then $t V \in \operatorname{dom}\left(e_{x}\right)$,

- if $h(x)<1$ then writing $x=(h, y) \in] 0,1\left[\times L, V=(\lambda, W) \in \mathbb{R} \times T_{y} L\right.$ under the identification $\left.X_{-}=\right] 0,1\left[h \times L\right.$, we have $\kappa(h, t) W \in \operatorname{dom}\left(e_{y}^{L}\right)$ and $(h, \lambda, t, u) \in \mathcal{V}$.

We define a injective map $E_{\mathcal{H}}: \mathcal{U} \rightarrow \mathcal{H}$ by setting:

- for $(x, V) \in T X^{o}$ with $h(x) \geqslant 1$ :

$$
E_{\mathcal{H}}(x, V, t, u)=\left\{\begin{array}{cc}
\left(x, e_{x}(t V), t, u\right) & \text { if } t>0 \\
(x, W, 0, u) & \text { if } t=0
\end{array}\right.
$$

- for $(x, V) \in T X^{o}$ with $h(x)<1$ and $x=(h, y), V=(\lambda, W)$ as above:

$$
E_{\mathcal{H}}(h, y, \lambda, W, t, u)=\left\{\begin{array}{cc}
\left(h, y, \lambda, e_{y}^{L}(\tau(h) W), 0,0\right) & \text { if }(t, u)=(0,0) \\
\left(h, y, l^{-1}(l(h)+\mu(h, t, u) \lambda), e_{y}^{L}(\kappa(h, t) W), t, u\right) & \text { if }(t, u) \neq(0,0)
\end{array}\right.
$$

One can check that $\mathcal{U}_{D}=E_{\mathcal{H}}^{-1}\left(\left.\mathcal{H}\right|_{D}\right)$ is an open subset of $\mathcal{U}$. We provide $\left.\mathcal{H}\right|_{D} ^{\prime}=\left.\mathcal{H}\right|_{D} \cap$ $E_{\mathcal{H}}(\mathcal{U})$ with the smooth structure such that the map : $E_{\mathcal{H}}:\left.\mathcal{U}_{D} \rightarrow \mathcal{H}\right|_{D} ^{\prime}$ is a diffeomorphism. On the other hand, $\left.\mathcal{H}\right|_{D} ^{\prime \prime}=\left.\mathcal{H}\right|_{D} \cap\left(\left.\left.\left.\mathcal{H}\right|_{A} \cup \mathcal{H}\right|_{B} \cup \mathcal{H}\right|_{C}\right)$ is open in $\left.\left.\left.\mathcal{H}\right|_{A} \cup \mathcal{H}\right|_{B} \cup \mathcal{H}\right|_{C}$ so it inherits a smooth structure as a submanifold of $\left.\left.\left.\mathcal{H}\right|_{A} \cup \mathcal{H}\right|_{B} \cup \mathcal{H}\right|_{C}$. The smooth structure given to $\left.\mathcal{H}\right|_{D} ^{\prime}$ and $\left.\mathcal{H}\right|_{D} ^{\prime \prime}$ are compatible and cover $\left.\mathcal{H}\right|_{D}$. The resulting smooth structure of $\left.\mathcal{H}\right|_{D}$ is compatible with the ones given to the three other subgroupoids, so we have given to $\mathcal{H}$ a smooth structure for which $E_{\mathcal{H}}$ is an exponential map.

Remark 1 1. The gluing function $l$ is important in the description of the Poincaré duals of elliptic noncommutative symbols. We will see that choosing $l=\log$ near $h=0$ leads to this description with the help of b-calculus, while choosing $l(h)=-1 / h$ near $h=0$ would lead to c-calculus. Different choices of $l$ produce different but diffeomorphic smooth structures on $\mathcal{H}$. Indeed, if $l, m$ are two such choices, the map $\mathcal{H} \rightarrow \mathcal{H}$ equal to identity if $(t, u) \neq(0,0)$ or $h \geqslant 1$ and sending $(h, \lambda, x, y, 0,0)$ to $\left(h, \lambda \cdot \frac{m^{\prime}(h)}{l^{\prime}(h)}, x, y, 0\right)$ is a smooth isomorphism between $\mathcal{H}$ with the smooth structure given by $l$ and $\mathcal{H}$ with the smooth structure given by $m$. This follows from a simple but tedious calculation and the fact that for any smooth function $f: \mathbb{R} \rightarrow \mathbb{R}$, the map:

$$
\phi: \mathbb{R}^{3} \rightarrow \mathbb{R} ;(x, \mu, \lambda) \mapsto \begin{cases}\frac{f(x)-f(x-\mu \lambda)}{\mu} & \text { if } \mu \neq 0 \\ \lambda f^{\prime}(x) & \text { if } \mu=0\end{cases}
$$

is smooth on $\mathbb{R}^{3}$. 
2. All other gluing functions are technical ingredients and their choice has no incidences on the desired description.

3. All subgroupoids listed in (1.12) inherit smooth structures and exponential maps from those of $\mathcal{H}$.

We will often use $\mathcal{G}^{q}=\left.\mathcal{H}\right|_{u=0}$ in the sequel. Using $E_{\mathcal{H}}$, we get an exponential map for this groupoid:

- for $\left.(x, V, t) \in \mathcal{U}\right|_{u=0}$ with $h(x) \geqslant 1$ :

$$
E_{\mathcal{G}^{q}}(x, V, t)=\left\{\begin{array}{cc}
\left(x, e_{x}(t V), t, u\right) & \text { if } t>0 \\
(x, W, 0, u) & \text { if } t=0
\end{array}\right.
$$

- for $\left.(x, V, t) \in \mathcal{U}\right|_{u=0}$ with $h(x)<1$ and $x=(h, y), V=(\lambda, W)$ :

$$
E_{\mathcal{G}^{q}}(h, y, \lambda, W, t)=\left\{\begin{array}{cc}
\left(h, y, \lambda, e_{y}^{L}(\tau(h) W), 0,0\right) & \text { if } t=0 \\
\left(h, y, l^{-1}(l(h)+t \lambda), e_{y}^{L}(\kappa(h, t) W), t, u\right) & \text { if } t>0
\end{array}\right.
$$

where we have replaced $\mu(h, t, 0)$ by $t$ to simplify.

The inverse of the exponential map $E_{\mathcal{G} q}$ will be noted shortly $\Theta$.

The following define a Haar system for $\mathcal{G}^{q}$ which is necessary to define in a convenient way its $C^{*}$-algebra:

$$
\begin{array}{ccc}
t>0, & \mathcal{G}_{(x, t)}^{q}=X^{o} \times\{t\}, & d \lambda^{(x, t)}\left(x^{\prime}\right)=\frac{l^{\prime}\left(h^{\prime}\right)}{t \tau\left(t, h^{\prime}\right)^{n}} d \mu_{x^{\prime}}=d \lambda^{t}\left(h^{\prime}=h\left(x^{\prime}\right)\right) \\
t=0 \text { and } h<1, & \mathcal{G}_{(h, y, 0)}^{q}=\mathbb{R} \times L \times\{0\}, & d \lambda^{(h, y, 0)}=\frac{1}{\tau(h)^{n}} d \mu^{\mathbb{R}} d \mu^{L}=d \lambda^{h, 0} \\
t=0 \text { and } h \geqslant 1, & \mathcal{G}_{(x, 0)}^{q}=T_{x} X^{o}, & d \lambda^{(x, 0)}=d \nu_{x}=d \lambda^{x}
\end{array}
$$

Remark that $d \lambda^{1}$ is equal to $\frac{1}{h} d \mu$ near $h=0$, in other words it coincides with the density coming from a $b$-metric like:

$$
g_{b}(h, y)=\frac{d h^{2}}{h^{2}}+g_{L}(y)
$$

\section{Equivalences of tangent spaces and Dirac elements}

\subsection{Two equivalent tangent spaces}

The main results of [7] are the construction of a Dirac element $D^{c} \in K K\left(T^{c} X \times X, \cdot\right)$, a dual Dirac element $\lambda^{c} \in K K\left(\cdot, T^{c} X \times X\right)$, where $\cdot$ stands for a point space, and the computations in bivariant $K$-theory:

$$
\lambda^{c} \underset{T^{c} X}{\otimes} D^{c}=1 \in K K(X, X) \text { and } \lambda^{c} \underset{X}{\otimes} D^{c}=1 \in K K\left(T^{c} X, T^{c} X\right)
$$

which give in particular the isomorphism

$$
\Sigma^{c}=\left(\lambda^{c} \underset{X}{\otimes} \cdot\right)=\left(. \underset{T^{c} X}{\otimes} D^{c}\right)^{-1}
$$

in (1.1). In this paper, we will prefer to work with $T^{q} X$ rather than $T^{c} X$, because the analog for $T^{q} X$ of $\Sigma^{c}$ :

$$
\Sigma^{q}=\left(\lambda^{q} \underset{X}{\otimes} \cdot\right)=\left(. \underset{T^{q} X}{\otimes} D^{q}\right)^{-1}: K_{0}(X) \stackrel{\simeq}{\longrightarrow} K^{0}\left(T^{q} X\right)
$$

has a nice description. We are going to describe the $K K$-equivalence between $T^{q} X$ and $T^{c} X$ in order to have a correct representant of $D^{q}$. 
Proposition 1 The deformation element $\partial_{H} \in K K\left(T^{q} X, T^{c} X\right)$ associated with $H$ in (1.10) is a KK-equivalence.

Proof : Let $\mathrm{ev}_{+}: H \rightarrow T X_{+} \times[0,1]$ be the restriction morphism and consider the commutative diagram:

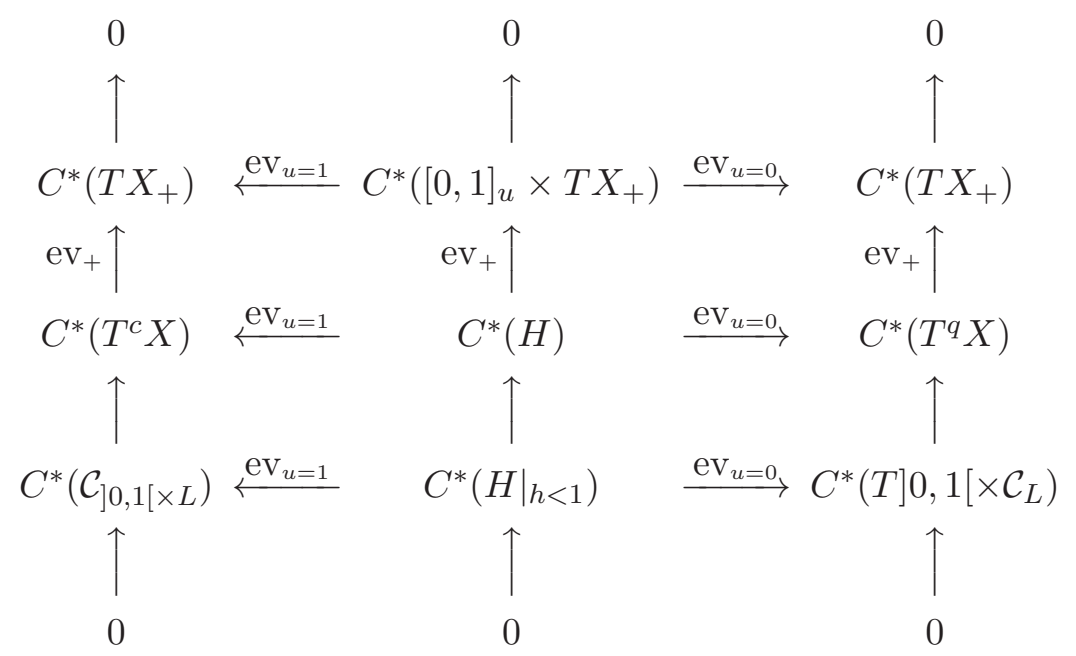

The columns are exact. On the top line the induced maps in $K$-theory provide

$$
\left[\mathrm{ev}_{u=0}\right]^{-1} \otimes\left[\mathrm{ev}_{u=1}\right]=1 \in K K\left(T X_{+}, T X_{+}\right)
$$

Observe that

$$
\left.\left.\left.\left.\left.H\right|_{h<1}=T\right] 0,1\left[\times \mathcal{C}_{L} \times\{0\} \cup \mathcal{C}_{] 0,1[\times L} \times\right] 0,1\right] \simeq(T] 0,1\left[\times\{0\} \cup \mathcal{C}_{] 0,1[} \times\right] 0,1\right]_{u}\right) \times \mathcal{C}_{L},
$$

and that $\left.\mathcal{G}_{0,1[}=T\right] 0,1\left[\times\{0\} \cup \mathcal{C}_{0,1}[\times] 0,1\right]$ is the usual tangent groupoid of the manifold ]0,1[. The associated Kasparov element

$$
\partial_{\mathcal{G}_{0,1[}} \in K K(T] 0,1\left[, \mathcal{C}_{0,1[}\right) \simeq K K\left(C_{0}\left(\mathbb{R}^{2}\right), \mathbb{C}\right) \simeq \mathbb{Z}
$$

is invertible with inverse given by the Bott generator of $K K\left(\mathbb{C}, C_{0}\left(\mathbb{R}^{2}\right)\right)$. It follows that in the bottom line

$$
\left[\mathrm{ev}_{u=0}\right]^{-1} \otimes\left[\mathrm{ev}_{u=1}\right]=\mathbf{s}_{C^{*}\left(\mathcal{C}_{L}\right)}\left(\partial_{\mathcal{G}_{] 0,1}}\right) \in K K(T] 0,1\left[\times \mathcal{C}_{L}, \mathcal{C}_{] 0,1[} \times \mathcal{C}_{L}\right)
$$

is invertible. Here $\mathbf{s}_{A}: K K(B, C) \rightarrow K K(B \otimes A, C \otimes A)$ is the usual tensorisation operation in Kasparov theory.

In particular the Kasparov elements $\left[\mathrm{ev}_{u=1}\right]$ in (2.4) and (2.6) are invertible. Hence, applying the five lemma to the long exact sequences in $K K$-theory associated with the first two columns of (2.3) give the invertibility of the element $\left[\mathrm{ev}_{u=1}\right]$ in the middle line of (2.3). This yields that $\partial_{H}$, equal to $\left[\mathrm{ev}_{u=0}\right]^{-1} \otimes\left[\mathrm{ev}_{u=1}\right]$ in the middle line, is invertible.

\subsection{The Dirac element for the ' $q$ ' version}

Let us turn to the description of $D^{q}$. We define:

$$
D^{q}:=\partial_{H} \underset{T^{c} X}{\otimes} D^{c} \in K K\left(T^{q} X \times X, \cdot\right)
$$

We recall the construction of $D^{c}$. We set:

$$
\partial^{c}:=\partial_{\mathcal{G}^{c}} \otimes \nu \in K K\left(T^{c} X, \cdot\right)
$$


where $\nu$ is the Morita equivalence $\nu \in K K\left(\mathcal{C}_{X^{o}}, \cdot\right)$ given by:

$$
\nu=\left(L^{2}\left(X^{o}\right), m, 0\right) \in K K\left(\mathcal{C}_{X^{o}}, \cdot\right)
$$

$\partial^{c}$ is called the pre-Dirac element and the Dirac element is

$$
D^{c}:=\Phi^{c} \otimes \partial^{c} \in K K\left(T^{c} X \times X, \cdot\right)
$$

where $\Phi^{c}$ is the $K K$-element associated to the map defined by

$$
\begin{aligned}
& \Phi^{c}: C^{*}\left(T^{c} X\right) \otimes C(X) \longrightarrow C^{*}\left(T^{c} X\right) \\
& a \otimes f \quad \longmapsto \quad a . f \circ \pi^{c}
\end{aligned}
$$

and $\pi^{c}$ is the composition of the range map of $T^{c} X$ with the projection $X^{o} \rightarrow X^{o} / \overline{X_{-}} \simeq X$. In the 'q' version we set:

$$
\partial^{q}=\partial_{\mathcal{G}^{q}} \otimes \nu
$$

and

$$
\begin{array}{cc}
\Phi^{q}: C^{*}\left(T^{q} X\right) \otimes C(X) & \longrightarrow C^{*}\left(T^{q} X\right) \\
a \otimes f & \longmapsto a . f \circ \pi^{q}
\end{array}
$$

where $\pi^{q}$ projects $T^{q} X$ onto $X$ like $\pi^{c}$ does $T^{c} X$ on $X$. We check that :

Proposition 2 The following equality holds:

$$
D^{q}=\Phi^{q} \otimes \partial^{q} \in K K\left(T^{q} X \times X, \cdot\right)
$$

where $D^{q}$ is defined by 2.7).

Proof : Let us consider the commutative diagram:

$$
\begin{array}{cccc}
C^{*}\left(\mathcal{C}_{X^{o}}\right) & \stackrel{\mathrm{ev}_{t=1}}{\longleftarrow} C^{*}\left(\mathcal{G}^{q}\right) & \stackrel{\mathrm{ev}_{t=0}}{\longrightarrow} & C^{*}\left(T^{q} X\right) \\
\mathrm{ev}_{u=0} \uparrow & \mathrm{ev}_{u=0} \uparrow & \mathrm{ev}_{u=0} \uparrow \\
C^{*}\left(\mathcal{C}_{X^{o}} \times[0,1]\right) & \stackrel{\mathrm{ev}_{t=1}}{\longleftarrow} C^{*}(\mathcal{H}) & \stackrel{\mathrm{ev}_{t=0}}{\longrightarrow} & C^{*}(H) \\
\mathrm{ev}_{u=1} \downarrow & \mathrm{ev}_{u=1} \downarrow & \mathrm{ev}_{u=1} \downarrow \\
C^{*}\left(\mathcal{C}_{X^{o}}\right) & \stackrel{\mathrm{ev}_{t=1}}{\longleftarrow} C^{*}\left(\mathcal{G}^{c}\right) & \stackrel{\mathrm{ev}_{t=0}}{\longrightarrow} & C^{*}\left(T^{c} X\right)
\end{array}
$$

At the level of $K K$-theory, the bottom line of the diagram gives $\partial^{q}$, up to the Morita equivalence $\nu$, while the top line gives $\partial^{c}$ (up to $\nu$ ). The right column gives the $K K$ equivalence $\partial_{H}$ while the product $\mathrm{ev}_{u=0}^{-1} \otimes \mathrm{ev}_{u=1}$ in the left column is obviously 1 in $K K\left(\mathcal{C}_{X^{o}}, \mathcal{C}_{X^{o}}\right)$. This gives:

$$
\partial_{H} \otimes \partial^{c}=\partial^{q} .
$$

To finish, let us introduce the multiplication morphism:

$$
\Phi^{H}: C^{*}(H) \otimes C(X) \rightarrow C^{*}(H)
$$

given by $\Phi^{H}(a, f)(\gamma)=a(\gamma) f\left(\pi^{H}(\gamma)\right)$, where the projection map $\pi^{H}: H \rightarrow X$ extends $\pi^{q}$ and $\pi^{c}$ in the obvious way. Denoting in the same way the restriction morphisms for the product groupoid $H \times X$, we get the formulas:

$$
\begin{gathered}
\mathbf{s}_{C(X)} \partial_{H}=\mathbf{s}_{C(X)}\left(\left[\mathrm{ev}_{u=0}\right]^{-1} \otimes\left[\mathrm{ev}_{u=1}\right]\right)=\left[\mathrm{ev}_{u=0}\right]^{-1} \otimes\left[\mathrm{ev}_{u=1}\right], \\
{\left[\mathrm{ev}_{u=1}\right] \otimes \Phi^{c}=\Phi^{H} \otimes\left[\mathrm{ev}_{u=1}\right] \quad ; \quad\left[\mathrm{ev}_{u=0}\right]^{-1} \otimes \Phi^{H}=\Phi^{q} \otimes\left[\mathrm{ev}_{u=0}\right]^{-1} .}
\end{gathered}
$$

Hence:

$$
\begin{aligned}
D^{q}=\partial_{H} \underset{T^{c} X}{\otimes} D^{c} & =\mathbf{s}_{C(X)}\left(\partial_{H}\right) \otimes D^{c}=\left[\mathrm{ev}_{u=0}\right]^{-1} \otimes\left[\mathrm{ev}_{u=1}\right] \otimes \Phi^{c} \otimes \partial_{X}^{c} \\
& =\Phi^{q} \otimes\left[\mathrm{ev}_{u=0}\right]^{-1} \otimes\left[\mathrm{ev}_{u=1}\right] \otimes \partial^{c}=\Phi^{q} \otimes \partial^{q}
\end{aligned}
$$




\section{Cycles of the $K$-theory of the tangent space}

\subsection{Symbols on a manifold as operators on the tangent space}

To proceed, we need some definitions about pseudodifferential calculus on groupoids. The notions summed up below can be found with full details in the litterature: [28, 18, 21, 6].

Let $G$ be a smooth groupoid (the space of units is allowed to be a manifold with boundary, but the fibers are manifolds without boundary). Let $U_{\gamma}: C^{\infty}\left(G_{s(\gamma)}\right) \rightarrow C^{\infty}\left(G_{r(\gamma)}\right)$ be the isomorphism induced by right multiplication: $U_{\gamma} f\left(\gamma^{\prime}\right)=f\left(\gamma^{\prime} \gamma\right)$. A linear operator $P: C_{c}^{\infty}(G) \rightarrow C^{\infty}(G)$ is a $G$-operator if there exists a family $P_{x}: C_{c}^{\infty}\left(G_{x}\right) \rightarrow C^{\infty}\left(G_{x}\right)$ such that $P(f)(\gamma)=P_{s(\gamma)}\left(\left.f\right|_{G_{s(\gamma)}}\right)(\gamma)$ and $U_{\gamma} P_{s(\gamma)}=P_{r(\gamma)} U_{\gamma}$.

A $G$-operator $P$ is a pseudodifferential operator on $G$ (resp. of order $m$ ) if for any open local chart $\Phi: \Omega \rightarrow s(\Omega) \times W$ of $G$ such that $s=p r_{1} \circ \Phi$ (that is, for any distinguished chart) and any cut-off function $\chi \in C_{c}^{\infty}(\Omega)$, we have $\left(\Phi^{*}\right)^{-1}(\chi P \chi)_{x} \Phi^{*}=a\left(x, w, D_{w}\right)$ where $a(x, w, \xi) \in S^{*}\left(s(\Omega) \times T^{*} W\right)$ is a classical symbol (resp. of order $\left.m\right)$.

One says that $P$ has support in $K \subset G$ if $\operatorname{supp}(P f) \subset K \cdot \operatorname{supp}(f)$ for all $f \in C_{c}^{\infty}(G)$.

These definitions extend immediately to the case of operators acting between sections of bundles on $G^{(0)}$ pulled back to $G$ with the range map $r$. The space of compactly supported pseudodifferential operators on $G$ acting on sections of $r^{*} E$ and taking values in sections of $r^{*} F$ will be noted $\Psi_{c}^{*}(G, E, F)$. If $F=E$ we get an algebra denoted by $\Psi_{c}^{*}(G, E)$.

Basic examples of the usefulness of these operators are the case of foliations [6, 28] and manifolds with corners [16]. This calculus is also used in [7] to define $K K$-theory classes and to compute some Kasparov products. Here, to motivate our definition of noncommutative symbols on a singular manifold, we explain in more details what is suggested in the introduction.

Let $V$ be a smooth compact riemannian manifold, $E$ a smooth vector bundle over $V$ and consider the tangent space $T V$ as a smooth groupoid (thus $r$ and $s$ are equal to the canonical projection map $T V \rightarrow V$ ).

Let $a \in \Psi_{c}^{*}(T V, E)$. By definition, $a$ is a smooth family $\left(a_{x}\right)_{x \in V}$ where $a_{x}$ is a translation invariant pseudodifferential operators on $T_{x} V$ (with coefficients in End $E_{x}$ ) and thus can be regarded as a distribution $a_{x}(X)$ on $T_{x} V$ acting by convolution on $C_{c}^{\infty}\left(T_{x} V, E_{x}\right)$, so:

$$
u \in C_{c}^{\infty}(T V, E), \quad a(u)(x, X)=a * u(x, X)=\int_{Y \in T_{x} V} a(x, X-Y) u(Y) d Y
$$

where the last integral is understood in the distributional sense. The distribution $a_{x}(X)$ being compactly supported, it has a Fourier transform $\widehat{a_{x}}(\xi)$ which is just its symbol. The whole family $\left(\widehat{a_{x}}\right)_{x}$ identifies with a classical symbol on $V$ taking values in End $E$ ), that is, $\widehat{a} \in S^{*}(V$, End $E)$ and since the Fourier transform exchanges convolution with pointwise multiplication, we get an algebra homomorphism:

$$
\begin{array}{ccc}
\mathcal{F}: \Psi_{c}^{*}(T V, E) & \rightarrow & S^{*}(V, \text { End } E) \\
a & \longmapsto & \widehat{a}(x, \xi)
\end{array}
$$

which is obviously injective. Conversely, the inverse Fourier transform associates to any symbol $b(x, \xi) \in S^{*}(V$, End $E)$ a distribution $\stackrel{\vee}{b}(x, X)$ which, as a convolution operator, is a $T V$-pseudodifferential operator by the formula:

$$
u \in C_{c}^{\infty}(T V, E), \quad \stackrel{\vee}{b} * u(x, X)=\int_{T_{x} V \times T_{x}^{*} V} e^{i(X-Y) \cdot \xi} b(x, \xi) u(x, Y) d Y d \xi
$$


Moreover, introducing a smooth function $\phi(x, X)$ on $T V$ equal to 1 if $X=0$ and equal to 0 if $|X|>1$, we get:

$$
\stackrel{\vee}{a}=\phi . \stackrel{\vee}{a}+(1-\phi) \stackrel{\vee}{a} \in \Psi_{c}^{*}(T V, E)+\mathcal{S}(T V, \text { End } E)
$$

where $\mathcal{S}(T V$, End $E)$ stands for the (Schwartz) space of smooth sections whose partial derivatives are rapidly decaying in the fibers of $T V$.

Thus, enlarging $\Psi_{c}^{*}(T V, E)$ as follows:

$$
\Psi^{*}(T V, E):=\Psi_{c}^{*}(T V, E)+\mathcal{S}(T V, \text { End } E),
$$

the algebra monomorphism (3.1) extends to an algebra isomorphism

$$
\mathcal{F}: \Psi^{*}(T V, E) \longrightarrow S^{*}(V, \text { End } E)
$$

which preserves the filtrations. For a general discussion about the enlargement of spaces of compactly supported pseudodifferential operators by adding regularizing ones, see [28, 13.

One can then reformulate the classical description of the $K$-theory with compact supports of $T^{*} V$ :

Proposition 3 Every element in $K^{0}\left(T^{*} V\right) \simeq K^{0}(T V)$ has a representant of the following form:

$$
[a]=\left(C^{*}(T V, E \oplus F),\left(\begin{array}{ll}
0 & a \\
b & 0
\end{array}\right)\right) \in K K(\cdot, T V)
$$

where $E, F$ are smooth vector bundles over $V$ and $a \in \Psi^{0}(T V, E, F), b \in \Psi^{0}(T V, F, E)$ satisfy $b a-1 \in \Psi^{-1}(T V, E), a b-1 \in \Psi^{-1}(T V, F)$.

\subsection{Noncommutative symbols and their ellipticity on a conical pseudo- manifold}

Motivated by the previous approach, we enlarge the space of compactly supported pseudodifferential operators on $T^{q} X$ and define them as noncommutative symbols on the pseudomanifold $X$. Definitions are given in the scalar case since the presence of vector bundles bring no issues. We introduce:

$$
\overline{T^{q} X}=\{0\} \times \mathbb{R} \times \mathcal{C}_{L} \cup T^{q} X=T\left[0,1\left[_{h} \times \mathcal{C}_{L} \cup T X_{+} \rightrightarrows M=\overline{X^{o}}\right.\right.
$$

Definition 1 Let $\tau$ be the function choosed in (1.15) and define the function $||:. T^{q} X \rightarrow$ $\mathbb{R}_{+}$by:

$$
|\gamma|= \begin{cases}\sqrt{\left(\frac{\operatorname{dist}_{L}(x, y)}{\tau(h)}\right)^{2}+\lambda^{2}} & \text { if } \gamma=(h, \lambda, x, y) \in T] 0,1\left[\times \mathcal{C}_{L}(i e, h<1)\right. \\ \sqrt{g_{x}(X, X)} & \text { if } \gamma=(x, X) \in T X_{+}(i e, h \geqslant 1)\end{cases}
$$

The restriction at $t=0$ of the Haar system $\mathcal{G}^{q}$ defined in (1.25) provides a Haar system for $T^{q} X$, and extended at $h=0$ in the obvious way, we get a Haar system for $\overline{T^{q} X}$. It is then easy to check that $|$.$| is a length function with polynomial growth on \overline{T^{q} X}$ and the corresponding Shwartz algebra is denoted by $\mathcal{S}\left(\overline{T^{q} X}\right)([13])$. Using the seminorms:

$$
p_{D, N}(f)=\sup _{\gamma \in T^{q} X}(1+|\gamma|)^{N}|D f(\gamma)|,
$$

where $N$ is a positive integer and $D \in \operatorname{Diff}\left(\overline{T^{q} X}\right)$ is a differential operator on $\overline{T^{q} X}$, this Schwartz algebra can be presented as follows:

$$
\mathcal{S}\left(\overline{T^{q} X}\right)=\left\{f \in C^{\infty}\left(\overline{T^{q} X}\right) \mid p_{D, N}(f)<+\infty \forall N \in \mathbb{N}, \forall D \in \operatorname{Diff}\left(\overline{T^{q} X}\right)\right\}
$$

By restriction at $h=0$ of these functions, we get the Schwartz algebra $\mathcal{S}\left(\mathbb{R} \times \mathcal{C}_{L}\right)$ of the groupoid $\mathbb{R} \times \mathcal{C}_{L}$ endowed with the restricted Haar system. 
Definition 2 The algebra of noncommutative symbols on $X$ is defined by:

$$
S^{*}(X)=\Psi_{c}^{*}\left(\overline{T^{q} X}\right)+\mathcal{S}\left(\overline{T^{q} X}\right) \subset \Psi^{*}\left(\overline{T^{q} X}\right)
$$

We also define $S_{0}^{*}(X)$ as the kernel of the restriction homomorphism at $h=0$ :

$$
\begin{array}{cc}
\rho: S^{*}(X) & \rightarrow \\
a & \left.\longmapsto a\right|_{h=0}
\end{array} \Psi_{c}^{*}\left(\mathbb{R} \times \mathcal{C}_{L}\right)+\mathcal{S}\left(\mathbb{R} \times \mathcal{C}_{L}\right)
$$

\section{Remark 2}

(a) The image of $\rho$ is exactly the algebra $\mathcal{P}_{\text {inv }}^{*}(\mathbb{R} \times L)$ of translation invariant pseudodifferential operators on $\mathbb{R} \times L$ defined by $R$. Melrose in [15].

(b) The smoothness of noncommutative symbols up to $h=0$ can be relaxed and singular behaviors can be of interest, see paragraph 3.4 .

The following observations will lead to the notion of ellipticity for these noncommutative symbols.

Proposition 4 The following inclusion holds

$$
S^{0}(X) \subset \mathcal{M}\left(C^{*}\left(T^{q} X\right)\right) .
$$

Moreover, we have $S^{-1}(X) \subset C^{*}\left(\overline{T^{q} X}\right)$ and

$$
S_{0}^{-1}(X)=S^{-1}(X) \cap C^{*}\left(T^{q} X\right) .
$$

Proof : It is known from [18, 28] that $\Psi^{0}\left(\overline{T^{q} X}\right) \subset \mathcal{M}\left(C^{*}\left(\overline{T^{q} X}\right)\right)$ and $\Psi^{-1}\left(\overline{T^{q} X}\right) \subset$ $C^{*}\left(\overline{T^{q} X}\right)$. Since $C^{*}\left(T^{q} X\right)$ is an ideal of $C^{*}\left(\overline{T^{q} X}\right)$ and since any $a \in \Psi^{0}\left(\overline{T^{q} X}\right)$ maps $C^{*}\left(T^{q} X\right)$ to itself, (3.6) is true. Since $C^{*}\left(T^{q} X\right)$ is the kernel of the restriction morphism $C^{*}\left(\overline{T^{q} X}\right) \rightarrow C^{*}\left(\mathbb{R} \times \mathcal{C}_{L}\right)$ at $h=0,(3.7)$ is obvious.

In the sequel, the algebra of small $b$-calculus [14] will be denoted by $\mathcal{P}_{b}^{*}(M)$ and its ideal consisting of operators with vanishing indicial families will be denoted by $\mathcal{P}_{b, 0}^{*}(M)$. Given a $\mathcal{G}^{q}$-pseudodifferential operator $P$, its restriction $\left.P\right|_{t}$ at any $t>0$ is a $\mathcal{C}_{X^{o}}$-pseudodifferential operator, that is, an ordinary pseudodifferential operator on the (open) manifold $X^{o}$. In fact, we will denote by $\Psi_{b}^{*}\left(\mathcal{G}^{q}\right)$ the algebra of $\mathcal{G}^{q}$-pseudodifferential operators whose restrictions $\left.P\right|_{t}$ at any $t>0$ are in the $b$-calculus of $M=\overline{X^{o}}$, that is, such that for all $t>0,\left.P\right|_{t} \in \mathcal{P}_{b}^{*}(M)$. The ideal of operators $P \in \Psi_{b}^{*}\left(\mathcal{G}^{q}\right)$ such that $\left.P\right|_{t} \in \mathcal{P}_{b, 0}^{*}(M)$ for all $t>0$ will be denoted by $\Psi_{b, 0}^{*}\left(\mathcal{G}^{q}\right)$. The previous proposition extends to these spaces:

Proposition 5 The following inclusions hold:

$$
\begin{gathered}
\Psi_{b}^{0}\left(\mathcal{G}^{q}\right) \subset \mathcal{M}\left(C^{*}\left(\mathcal{G}^{q}\right)\right) \\
\Psi_{b, 0}^{-1}\left(\mathcal{G}^{q}\right) \subset C^{*}\left(\mathcal{G}^{q}\right)
\end{gathered}
$$

This follows from properties of $b$-calculus and the proposition 4 .

Definition 3 A noncommutative symbol $a \in S^{*}(X)$ is elliptic if it is invertible in $S^{*}(X)$ modulo $S_{0}^{-1}(X)$.

A noncommutative symbol $a \in S^{*}(X)$ is relatively elliptic if it is invertible in $S^{*}(X)$ modulo $S^{-1}(X)$.

The relative ellipticity of $a \in S^{*}(X)$ is exactly its ellipticity as a pseudodifferential operator on $\overline{T^{q} X}$. The notion of ellipticity for our noncommutative symbols is stronger and is similar to the notion of full ellipticity [14, 17].

Indeed, let $\sigma(a) \in C^{\infty}\left(S^{*} M\right)$ be the principal symbol of $a \in S^{*}(X)$ viewed as a pseudodifferential operator on $\overline{T^{q} X}$. We call $(\sigma(a), \rho(a)) \in C^{\infty}\left(S^{*} M\right) \times \mathcal{P}_{i n v}^{*}(\mathbb{R} \times L)$ the leading part of $a$. 
Proposition 6 The following assertions are equivalent:

1. The noncommutative symbol a is elliptic on $X$.

2. The leading part of a is invertible.

Proof : (i) $\Rightarrow$ (ii) is obvious. Conversely, let $a \in \Psi^{d}\left(\overline{T^{q} X}\right)$ be a noncommutative symbol whose principal part is invertible. Since $a$ is an elliptic $\overline{T^{q} X}$-pseudodifferential operator, we can choose $\widetilde{b} \in \Psi^{-d}\left(\overline{T^{q} X}\right)$ inverting $a$ modulo $\Psi^{-1}\left(\overline{T^{q} X}\right)$. From the smoothness of the family $a$ we get a continuous map $\left.h \in[0,1] \mapsto a\right|_{h} \in \mathcal{P}_{i n v}^{*}(\mathbb{R} \times L)$ and the invertibility of $\rho(a)$ implies the invertibility of $\left.a\right|_{h}$ if $h<\alpha$ for some $\alpha>0$. We pick a cut-off function $\omega \in C_{c}^{\infty}[0, \alpha[$ such that $\omega(0)=1$ and we set :

$$
b=\omega\left(\left.a\right|_{h}\right)^{-1}+(1-\omega) \widetilde{b}
$$

Then

$$
a b=\omega+(1-\omega) a \widetilde{b}=\omega+(1-\omega)(1+q)=1+(1-\omega) q,
$$

where $q \in \Psi^{-1}\left(\overline{T^{q} X}\right)$, and $a b-1 \in S_{0}^{-1}(X)$ is proved. Things are similar for $b a-1$.

\section{3 $K$-theory of the tangent space}

We prove in this paragraph that elliptic noncommutative symbols, when vector bundles are allowed, are the cycles of $K^{0}\left(T^{q} X\right)$. As already quoted, definitions 1 and 2 extend immediately to the case of vector bundles and we note $S^{*}(X, E, F)$ the space of noncommutative symbols on $X$ acting between sections of bundles $E, F$ over $M$. With the convention $S^{*}(X, E):=S^{*}(X, E, E)$, the proposition 4 becomes:

$$
S^{0}(X, E) \subset \mathcal{L}\left(C^{*}\left(T^{q} X, E\right)\right), \quad S_{0}^{-1}(X, E) \subset \mathcal{K}\left(C^{*}\left(T^{q} X, E\right)\right)
$$

Therefore, we can associate to each elliptic noncommutative symbol $a \in S^{0}(X ; E, F)$ on $X$ of order 0 , an element in the $K$-theory of $T^{q} X$ :

Definition 4 Let $a \in S^{0}(X ; E, F)$ be an elliptic noncommutative symbol on $X$. We set:

$$
[a]:=\left[C^{*}\left(T^{q} X, E \oplus F\right), \mathbf{a}\right] \in K K\left(\cdot, T^{q} X\right) \simeq K^{0}\left(T^{q} X\right)
$$

where:

$$
\mathbf{a}=\left(\begin{array}{ll}
0 & b \\
a & 0
\end{array}\right)
$$

and $b$ is any noncommutative symbol inverting a modulo $S_{0}^{-1}$.

It is straightforward that $[a]$ does not depend on the choice of the quasi-inverse $b$. The main result of this section is that proposition 3 holds in this new framework:

Theorem 1 Every element of $K^{0}\left(T^{q} X\right)$ has a representant among elliptic noncommutative symbols. More precisely:

$$
K^{0}\left(T^{q} X\right)=\{[a] \mid a \text { is an elliptic noncommutative symbol on } X \text { of order } 0\},
$$

Remarks 1 1. Considering the Kasparov ungraded modules given by $\left(C^{*}\left(T^{q} X, E\right), a\right)$ where $a \in S^{0}(X, E)$ and $a^{2}-1 \in S_{0}^{-1}(X, E)$, the conclusion is the same for $K^{1}\left(T^{q} X\right)$.

2. In the same way, relative elliptic noncommutative symbols span the $K$-theory of $\overline{T^{q} X}$. Observe that $\overline{T^{q} X}$ is $K K$-equivalent to $T X^{o}$ which is $K K$-dual to $M=\overline{X^{o}}$. 
Proof of the theorem: Let us denote by $\Delta$ the following subset of $K^{0}\left(T^{q} X\right)$ :

$\{[a] \mid a$ is an elliptic noncommutative symbol on $X$ of order 0$\}$.

From the exact sequence of $C^{*}$-algebras:

$$
0 \rightarrow C^{*}\left(\mathcal{C}_{L} \times T I\right) \stackrel{i}{\rightarrow} C^{*}\left(T^{q} X\right) \stackrel{\mathrm{ev}_{+}}{\rightarrow} C^{*}\left(T X_{+}\right) \rightarrow 0
$$

we get the exactness of

$$
K^{0}\left(\mathcal{C}_{L} \times T I\right) \stackrel{i}{\rightarrow} K^{0}\left(T^{q} X\right) \stackrel{\mathrm{ev}_{+}}{\rightarrow} K^{0}\left(T X_{+}\right)
$$

If $i\left(K^{0}\left(\mathcal{C}_{L} \times T I\right)\right) \subset \Delta$ and $\mathrm{ev}_{+}\left(K^{0}\left(T^{q} X\right)\right) \subset \mathrm{ev}_{+}(\Delta)$ then the theorem is true. These inclusions are checked in the following lemmas.

Lemma 1 The inclusion $i\left(K^{0}\left(\mathcal{C}_{L} \times T I\right)\right) \subset \Delta$ holds.

Proof of the lemma: It is sufficient to find a generator $e$ of $K^{0}\left(C^{*}\left(\mathcal{C}_{L} \times T I\right)\right) \simeq \mathbb{Z}$ such that $i_{*}(e) \in \Delta$. We will define first an appropriate generator of $K_{0}\left(C_{0}\left(\mathbb{R}^{2}\right) \otimes \mathcal{K}\left(L^{2}(L)\right)\right)$ and then we will use an isomorphism $C^{*}\left(\mathcal{C}_{L} \times T I\right) \simeq C_{0}\left(\mathbb{R}^{2}\right) \otimes \mathcal{K}\left(L^{2}(L)\right)$,

Let us choose the following generator of $K_{0}\left(C_{0}\left(\mathbb{R}^{2}\right)\right)$ :

$$
x=[\mathcal{E}, 1, F] \text { where: } \mathcal{E}=C_{0}\left(\mathbb{R}^{2}\right) \oplus C_{0}\left(\mathbb{R}^{2}\right), F:=\frac{d}{\sqrt{1+d^{2}}} \text { and } d:=\left(\begin{array}{cc}
0 & h-i \lambda \\
h+i \lambda & 0
\end{array}\right) .
$$

On the other hand, let $B_{+}$be an elliptic pseudodifferential operator on $L$ with index 1 . Without loss of generality, we may assume that $B_{+}$is of order 1 , is almost unitary (ie, unitary modulo 0 order operators) and acts between sections of a trivial bundle $L \times \mathbb{C}^{k}$. Let $b_{+} \in C^{\infty}\left(S^{*} L, U_{k}(\mathbb{C})\right)$ be its principal symbol. Then the following represents $1 \in K^{0}\left(\mathcal{C}_{L}\right)$ :

$$
x^{\prime}=\left[\mathcal{E}^{\prime}, 1, F^{\prime}\right], \text { where: } \mathcal{E}^{\prime}=\mathcal{K}\left(\left(L^{2}\left(L ; \mathbb{C}^{k}\right)\right)^{2}\right), F^{\prime}:=\frac{B}{\sqrt{1+B^{2}}} \text { and } B:=\left(\begin{array}{cc}
0 & B_{+}^{*} \\
B_{+} & 0
\end{array}\right)
$$

Now the Kasparov product $x^{\prime \prime}=x \underset{\mathbb{C}}{\otimes} x^{\prime}$ is a generator of $K_{0}\left(C_{0}\left(\mathbb{R}^{2}\right) \otimes \mathcal{K}\left(L^{2}(L)\right)\right)$ and is represented by $\left(\mathcal{E}^{\prime \prime}, F^{\prime \prime}\right)$ where:

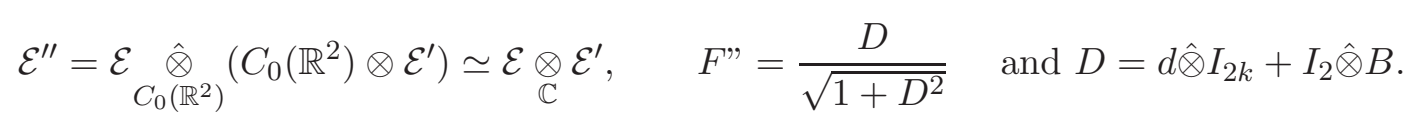

Here $I_{n}$ denotes the identity matrix of rank $n$ and $\hat{\otimes}$ is the graded tensor product. Recall the matricial expression of $D$ :

$$
D=d \hat{\otimes} I_{2 k}+I_{2} \hat{\otimes} B=\left(\begin{array}{cccc}
0 & 0 & 1 \otimes B_{+}^{*} & d_{-} \otimes 1 \\
0 & 0 & d_{+} \otimes 1 & -1 \otimes B_{+} \\
1 \otimes B_{+} & d_{-} \otimes 1 & 0 & 0 \\
d_{+} \otimes 1 & -1 \otimes B_{+}^{*} & 0 & 0
\end{array}\right)
$$

It is clear that $D$ is a pseudodifferential operator on $L$ with parameters $(h, \lambda) \in \mathbb{R}^{2}$ (of order 1 ) in the sense of [25], acting on the sections of the product bundle $L \times \mathbb{C}^{4 k}$. Following the construction of complex powers given in [25], we see that $F$ " remains in the same space of operators with parameters (but of course, it is of order 0). Let us find a better representant of $x^{\prime \prime}$ by trivializing $F^{\prime \prime}$ at $+\infty$. Let us introduce the matrix:

$$
J=\left(\begin{array}{cc}
0 & K \\
K & 0
\end{array}\right) \in M_{4 k}(\mathbb{C}) \text { where } K=\left(\begin{array}{cc}
0 & I_{k} \\
I_{k} & 0
\end{array}\right) \in M_{2 k}(\mathbb{C})
$$


We choose a smooth decreasing function $M$ equal to 1 on ] $-\infty, 0]$ and vanishing near $h=+\infty$. We set:

$$
C=M^{1 / 2} F^{\prime \prime}+(1-M)^{1 / 2} J .
$$

We are going to check that $C^{2}-1 \in \mathcal{K}\left(\mathcal{E}^{\prime \prime}\right)$. Observe that:

$C^{2}=M\left(F^{\prime \prime}\right)^{2}+1-M+[M(1-M)]^{1 / 2}\left[F^{\prime \prime}, J\right]=1+[M(1-M)]^{1 / 2}\left[F^{\prime \prime}, J\right] \bmod \mathcal{K}\left(\mathcal{E}^{\prime \prime}\right)$

where the bracket is $\mathbb{Z}_{2}$-graded. To compute $\left[F^{\prime \prime}, J\right]$, let us proove that $\left(1+D^{2}\right)^{-1 / 2}$ commutes with $J$. Setting :

$$
\Delta=1+D^{2}=\left(\begin{array}{cc}
\Delta_{+} & 0 \\
0 & \Delta_{-}
\end{array}\right)=\left(\begin{array}{cccc}
H_{+} & 0 & 0 & 0 \\
0 & H_{-} & 0 & 0 \\
0 & 0 & H_{-} & 0 \\
0 & 0 & 0 & H_{+}
\end{array}\right)
$$

one gets:

$$
\Delta J=\left(\begin{array}{cc}
0 & \Delta_{+} K \\
\Delta_{-} K & 0
\end{array}\right), \quad J \Delta=\left(\begin{array}{cc}
0 & K \Delta_{-} \\
K \Delta_{+} & 0
\end{array}\right)
$$

and:

$$
\Delta_{+} K=\left(\begin{array}{cc}
0 & H_{+} \\
H_{-} & 0
\end{array}\right)=K \Delta_{-}, \quad \Delta_{-} K=\left(\begin{array}{cc}
0 & H_{-} \\
H_{+} & 0
\end{array}\right)=K \Delta_{+},
$$

hence $J \Delta=\Delta J$ which implies, using functional calculus, that $J$ commutes with $\Delta^{-1 / 2}$, hence:

$$
\left[F^{\prime \prime}, J\right]=\Delta^{-1 / 2}(D J+J D)=\Delta^{-1 / 2}(2 h)=(2 h)\left(1+h^{2}+\lambda^{2}+I_{2} \otimes B^{2}\right)^{-1 / 2} .
$$

Since $h \mapsto M(h)(1-M(h))$ has compact support, we conclude that $[M(1-M)]^{1 / 2}\left[F^{\prime \prime}, J\right] \in$ $\mathcal{K}\left(\mathcal{E}^{\prime \prime}\right)$, hence $C^{2}=1 \bmod \mathcal{K}\left(\mathcal{E}^{\prime \prime}\right)$.

We thus get $\left[\mathcal{E}^{\prime \prime}, C\right] \in K_{0}\left(C_{0}\left(\mathbb{R}^{2}\right) \otimes \mathcal{K}\left(L^{2}(L)\right)\right)$ and $C_{t}=M_{t}^{1 / 2} F^{\prime \prime}+\left(1-M_{t}\right)^{1 / 2} J$ with $M_{t}(h)=M(t h)$ provides an operatorial homotopy between $x "=\left(\mathcal{E}^{\prime \prime}, F^{\prime \prime}\right)$ and $\left(\mathcal{E}^{\prime \prime}, C\right)$.

Using a Fourier transform with respect to the variable $\lambda$ and a reparametrization $\mathbb{R} \simeq] 0,1\left[\right.$ on $h$, we get an isomorphism $\phi: C_{0}\left(\mathbb{R}^{2}\right) \otimes \mathcal{K}\left(L^{2}(L)\right) \stackrel{\widetilde{\rightrightarrows}}{\rightarrow} C^{*}\left(\mathcal{C}_{L} \times T I\right)$ and $C$ gives rises to an element still noted $C$ and belonging to $\Psi^{0}\left(\mathcal{C}_{L} \times T I, \mathbb{C}^{4 k}\right)$. We now set

$$
e=\phi_{*}\left(x^{\prime \prime}\right)=\phi_{*}\left(\mathcal{E}^{\prime \prime}, C\right)=\left[C^{*}\left(\mathcal{C}_{L} \times T I, \mathbb{C}^{4 k}\right), C\right] \in K K\left(\cdot, \mathcal{C}_{L} \times T I\right)
$$

Finally we extend $C$ to $T^{q} X$ by setting $C=J$ on $T X_{+}$thanks to the formula (3.11). Hence:

$$
i_{*}(e)=\left[C^{*}\left(T^{q} X, \mathbb{C}^{4 k}\right), C\right] \in K K\left(\cdot, T^{q} X\right) \text { and } C \in \Psi^{0}\left(\overline{T^{q} X}, \mathbb{C}^{4 k}\right) \text {, hence } i_{*}(e) \in \Delta \text {. }
$$

Lemma 2 The equality $e v_{+}([\Delta])=K^{0}\left(T X_{+}\right)$holds.

Proof of the lemma: To each $K$-theory class $\sigma \in K^{0}\left(T^{*} X_{+}\right)$we shall associate $a_{\sigma} \in \Delta$ with $\left.\left(a_{\sigma}\right)\right|_{T X_{+}}=\sigma$.

Each element of $\sigma \in K^{0}\left(T^{*} X_{+}\right)$can be represented by a continuous section $f$ over $T^{*} X_{+} \backslash X_{+}$of the bundle Iso $\left(\pi^{*} E, \pi^{*} F\right)$ for some complex vector bundles $E, F$ over $X_{+}$ pulled-back by $\pi: T^{*} X_{+} \rightarrow X_{+}$. One can assume that $f$ is homogeneous of degre 0 in the fibers of $T^{*} X_{+}$and independent of $h$ near $\{h=1\}=\partial X_{+}$.

One sets $E=F=X_{+} \times \mathbb{C}$ since the general case is identical. Using the StoneWeierstrass theorem, we can find $g \in C^{\infty}\left(T^{*} X_{+}\right)$polynomial in $\xi$, independent of $h$ near 
$h=1$ and approximating uniformily $f$ in the corona $\left\{\xi \in T^{*} X_{+}|1 / 2 \leqslant| \xi \mid \leqslant 2\right\}$ up to an arbitrary small $\varepsilon>0$. Thus, modifying $f$ by:

$$
f(x, \xi)=g\left(x, \frac{\xi}{\sqrt{1+|\xi|^{2}}}\right)
$$

one gets another representant of $\sigma \in K^{0}\left(T^{*} X_{+}\right)$.

Choosing an exponential map $\theta$ for $\overline{T^{q} X}$, a cut-off function $\phi \in C_{c}^{\infty}\left(\overline{T^{q} X}\right)$ equal to 1 on units and extending $f$ to $T^{*} X^{o}$ in the obvious way, one can define the following noncommutative symbol on $X$ :

$$
a_{f}(u)(\gamma)=\operatorname{Op}_{\theta, \phi}(f)(u)(\gamma):=\int_{\xi \in T_{x}^{*} X^{o}, s\left(\gamma^{\prime}\right)=x^{\prime}} \underset{i<\theta^{-1}\left(\gamma^{\prime} \gamma^{-1}\right), \xi>}{ } f(x, \xi) \phi\left(\gamma^{\prime} \gamma^{-1}\right) u\left(\gamma^{\prime}\right) d \gamma^{\prime} d \xi
$$

where $x=r(\gamma), x^{\prime}=s(\gamma)$ and $u \in C_{c}^{\infty}\left(\overline{T^{q} X}\right)$. This noncommutative symbol is relatively elliptic on $X$, which is not sufficient here. We then consider the restriction $a_{0}$ of $a_{f}$ at $h=0$. If we note $\theta_{0}, \phi_{0}$ the corresponding restrictions of $\theta, \phi$, we have, using the same formula as (3.13):

$$
a_{0}=\mathrm{Op}_{\theta_{0}, \phi_{0}}\left(f_{0}\right) \in \mathcal{P}_{\text {inv }}^{0}(\mathbb{R} \times L) .
$$

Taking the Fourier transform with respect to the real variable in the above operator, we get a pseudodifferential operator $\widehat{a_{0}}(\lambda)$ on $L$ with parameter $\lambda \in \mathbb{R}$ which satisfies the condition of ellipticity with parameters, hence, by a classical result on operators with parameters, $\widehat{a_{0}}(\lambda)$ is invertible for large $|\lambda|$. Note that $\theta, \phi$ can be chosen so that:

$$
\theta_{0}: \mathbb{R} \times T L \ni(\lambda, y, V) \mapsto\left(\lambda, \exp _{y}^{L}(V)\right) \in \mathbb{R} \times \mathcal{C}_{L} \text { for small }|V| \text { and } \phi_{0}\left(\lambda, y, y^{\prime}\right)=\phi_{L}\left(y, y^{\prime}\right),
$$

where $\exp ^{L}$ is for instance the exponential map associated with the metric (1.5) and $\phi_{L}$ is compactly supported in the range of $\exp _{L}$ and satisfies $\phi_{L}(y, y)=1$. It follows that, writing $x=(h, y) \in[0,1] \times L ; \xi=(\lambda, \eta) \in T_{x}^{*} X_{+} \simeq \mathbb{R} \times T_{y}^{*} L$ and $f_{0}(x, \xi)=f_{0}(y, \lambda, \eta)$ :

$$
\widehat{a_{0}}(\lambda)(u)(y)=\int_{y^{\prime} \in L, \eta \in T_{y}^{*} L} e^{i<\left(\exp _{y}^{L}\right)^{-1}\left(y^{\prime}\right), \eta>} f_{0}(y, \lambda, \eta) \phi_{L}\left(y, y^{\prime}\right) u\left(y^{\prime}\right) d y^{\prime} d \eta
$$

where $u \in C^{\infty}(L)$ and $f_{0}$ denotes the restriction of $f$ at $h=0$.

Observe that $f_{0}$ has a holomorphic extension with respect to the cotangent variable $\lambda \in \mathbb{R}$ in the strip

$$
\mathcal{B}=\{z=\lambda+i u \in \mathbb{C} \mid-1 / 2<u<1 / 2\} .
$$

Indeed, the following function:

$$
f_{0}(y, z, \eta)=g\left(x, \frac{(z, \eta)}{\sqrt{1+z^{2}+|\eta|^{2}}}\right)
$$

makes sense as a holomorphic function in $z=\lambda+i u \in \mathcal{B}$ taking values in the space $C^{\infty}\left(T^{*} L\right)$ and is equal to $f_{0}$ when $u=0$. Moreover, for fixed $\left.u \in\right]-1 / 2,1 / 2[$, the function:

$$
(y, \lambda, \eta) \mapsto f_{0}(y, \lambda+i u, \eta)
$$

is a symbol of order 0 on $L$ with parameter $\lambda \in \mathbb{R}$ and one can find a constant $C$ independent of $y, \lambda, \eta$ such that:

$$
\left|f_{0}(y, \lambda+i u, \eta)-f_{0}(y, \lambda, \eta)\right| \leqslant C . u
$$

Since $f_{0}(y, \lambda, \eta)$ satisfies by construction the condition of ellipticity for symbols on $L$ with parameters $\lambda \in \mathbb{R}$, the previous estimate ensures that the same is true for $f_{0}(y, \lambda+i u, \eta)$ 
assuming that $|u|<\alpha$ for some $\alpha>0$ small enough. In the sequel we restrict the strip $\mathcal{B}$ according to this ellipticity condition.

It follows that (3.14) gives rise to a holomorphic family $z \mapsto \widehat{a_{0}}(z)$ taking values in elliptic pseudodifferential operators on $L$ of order 0 . We have noted earlier that there exists $z \in \mathcal{B}$ such that $\widehat{a_{0}}(z)$ is invertible, so by a classical result on homolorphic families of Fredholm operators, the sets:

$$
\left\{z=\lambda+i \rho|| \rho \mid \leqslant \alpha^{\prime} \text { and } p_{z} \text { is not invertible }\right\}
$$

are finite for all $\alpha^{\prime}<\alpha$. Hence, there exists $\beta$ such that $\widehat{a_{0}}(\lambda+i \beta)$ is invertible for all $\lambda \in \mathbb{R}$.

Observe also that each $\widehat{a_{0}}(z)$ restricted to horizontal lines $\operatorname{im}(z)=u$ in $\mathcal{B}$ is a pseudodifferential operator on $L$ with parameter $\lambda=\operatorname{re}(z)$, which allows to define $a_{u} \in \mathcal{P}_{i n v}^{*}(\mathbb{R} \times L)$ by:

$$
\widehat{a_{u}}(\lambda)=\widehat{a_{0}}(\lambda+i u)
$$

Choosing a smooth function $u(h)$ such that $u(0)=\beta$ and $u(1)=0$, we can define the required elliptic noncommutative symbol $a_{\sigma}$ on $X$ by:

$$
\left.a_{\sigma}\right|_{X_{+}}=a_{f} \text { and }\left.a_{\sigma}\right|_{h}=a_{u(h)} \text { for all } 0 \leqslant h \leqslant 1
$$

\subsection{Unbounded noncommutative symbols, Fuchs type noncommutative symbols}

We can also associate $K$-theory classes to elliptic noncommutative symbols on $X$ of positive order. To do that, we state:

Proposition 7 Let $a \in S^{m}(X, E)$ be an elliptic noncommutative symbol on $X$ with $m>0$. Let us consider a as an unbounded operator on $C^{*}\left(T^{q} X, E\right)$ with domain $C_{c}^{\infty}\left(T^{q} X, E\right)$. Then its closure $\bar{a}$ is regular ([2]).

Proof : The closure of $a$ with domain $C_{c}^{\infty}\left(\overline{T^{q} X}, E\right)$ is regular as an unbounded operator on $C^{*}\left(\overline{T^{q} X}, E\right)$ and following the proof of this result in [28, we see that everything remains true if we consider $a$ as an unbounded operator on $C^{*}\left(T^{q} X, E\right)$ with domain $C_{c}^{\infty}\left(T^{q} X, E\right)$. Note that the result still holds if $a$ is only relatively elliptic on $X$. As a consequence, to each elliptic noncommutative symbol $a$ on $X$ of order $m>0$ corresponds a morphism $q(a)=a\left(1+a^{*} a\right)^{-1 / 2} \in \mathcal{L}\left(C^{*}\left(T^{q} X, E\right)\right)$ and using the construction of complex powers given in [27, we get:

Proposition 8 Let $a \in S^{m}(X, E)$ be an elliptic noncommutative symbol of order $m>0$. 1) $\left(1+a^{*} a\right)^{-1 / 2}$ belongs to $S^{-m}(X, E)$.

2) $q(a)$ is an elliptic noncommutative symbol on $X$.

Proof : 1) Done in [27.

2) Let $b$ be a parametrix for $a$, that is $a b=1+r, b a=1+s$ with $r, s$ regularizing operators vanishing at $h=0$. Then $\left(1+a^{*} a\right)^{1 / 2} b$ is a parametrix for $q(a)$. Now we can associate to each elliptic $a \in S^{m}(X, E, F)$ the following $K$-theory class : $[q(a)]$. Note that these noncommutative symbols do not produce directly unbounded $K K$-theoritic elements ([2]) since $\left(1+a^{*} a\right)^{-1 / 2}$ is not a compact operator on the $C^{*}\left(T^{q} X\right)$-Hilbert module $C^{*}\left(T^{q} X, E\right)$. This defect leads us to consider Fuchs type noncommutative symbols. Let $\varphi$ be a positive smooth increasing function of $h$, equal to 1 if $h \geqslant 1$ and satisfying $\varphi(h)=h$ near $h=0$.

Definition 5 An element $p \in \Psi^{*}\left(T^{q} X, E\right)$ is a Fuchs type noncommutative symbol on $X$ if $\varphi^{l} p$ belongs to $S^{m}(X, E)$ for some $l \in \mathbb{R}_{+}$. The infimum of such $l$ is then called the fuchs type order of $p$. A Fuchs type noncommutative symbol $p$ with Fuchs type order $l$ strictly positive is elliptic if the noncommutative symbol $\varphi^{l} p$ is elliptic on $X$. 
For an elliptic Fuchs type noncommutative symbol $p$, we can define as before $\left(1+p^{*} p\right)^{-1 / 2} \in$ $\mathcal{L}\left(C^{*}\left(T^{q} X, E\right)\right)$. Thanks to the unbounded behavior of $p$ with respect to $h$ at $h=0$, the operator $\left(1+p^{*} p\right)^{-1 / 2}$ is actually compact so $\left(C^{*}\left(T^{q} X, E\right), p\right)$ provides an unbounded $K K$-theoritic element in the sense of [2].

Examples of such symbols come from Dirac type operators on $X$, where the latter is provided with a conical metric $g=d h^{2}+h^{2} g_{L}$, and their typical expression near $h=0$ is:

$$
p=h^{-1}\left(\begin{array}{cc}
0 & -\partial_{\lambda}+S \\
\partial_{\lambda}+S & 0
\end{array}\right)
$$

where $S$ is a Dirac type operator on $L$. See [8] for a developpement of this example.

\section{Poincaré dual of elliptic noncommutative symbols}

\subsection{Construction of a noncommutative symbol map}

We are going to define a noncommutative symbol map for $b$-operators using a deformation process encoded by $\mathcal{G}^{q}$. We then get a generalization of the complete symbol map for manifolds [10, 30], and like the notion that it generalizes, the noncommutative symbol is not canonical and depends on several choices: exponential maps, cut-off functions, connections on vector bundles. The idea of the construction is very close to [10, 9].

We motivate the forthcoming constructions by recalling the case of differential operators on a smooth manifold $V$. Let $Q$ be a differential operator on $V$ and:

$$
Q\left(x, D_{x}\right)=\sum_{\alpha} a_{\alpha}(x) D_{x}^{\alpha}
$$

its expression in a given local chart. For each $t \in] 0,1]$, the differential operator $P_{t}$ on $V$ defined locally by:

$$
P_{t}\left(x, D_{x}\right)=Q\left(x, t D_{x}\right)
$$

is well defined and setting:

$$
P_{0}\left(x, D_{X}\right)=\sum_{\alpha} a_{\alpha}(x) D_{X}^{\alpha} \in \operatorname{Diff}\left(T_{x} V\right),
$$

we get a differential operator $P=\left(P_{t}\right)_{t \in[0,1]}$ on the tangent groupoid $\mathcal{G}_{V}=T V \times\{0\} \cup$ $\left.\left.\mathcal{C}_{V} \times\right] 0,1\right]$ of $V$. As explained in paragraph [3.1, $P_{0}$ represents exactly the (total) symbol of $Q$.

Let us do the same thing for $b$-differential operators on $M=\overline{X^{\circ}}$ with the tangent groupoid $\mathcal{G}^{q}(1.9)$ of the pseudomanifold $X$.

From now on, the gluing function $l(1.18)$ is equal to logarithm function $l=\log$ near $h=0$. Let $Q$ be a $b$-differential operator on $M$ [14]. That means that near $\{h=0\}=\partial M$, one has, writing $x=(h, y) \in] 0,1[\times L$ :

$$
Q=\sum_{k} a_{k}\left(h, y, D_{y}\right)\left(h \partial_{h}\right)^{k}
$$

where $a_{k}$ are differential operators on $L$, depending smoothly in $h$. We define the family $\left(P_{t}\right)_{t \in[0,1]}$ as in (4.1), (4.2) on $X_{+}$, while we set on $X_{-}$, writing $X=(\lambda, V) \in \mathbb{R} \times T_{y} L$ :

$$
\begin{aligned}
& P_{t}=\sum_{k} a_{k}\left(h, y, \kappa(t, h) D_{y}\right)\left(\frac{t}{l^{\prime}(h)} \partial_{h}\right)^{k} \text { if } t>0, \\
& P_{0}=\sum_{k} a_{k}\left(h, y, \tau(h) D_{y}\right)\left(D_{\lambda}\right)^{k} \text { if } h<1 \text { if } t=0 .
\end{aligned}
$$


The functions $\kappa$ and $\tau$ are those chosen in (1.16) and (1.15). Observe that for $h$ close enough to 0 , (4.3) and (4.4) give:

$$
P_{t}=\sum_{k} a_{k}\left(h, y, D_{y}\right)\left(t h \partial_{h}\right)^{k} \text { and } P_{0}=\sum_{k} a_{k}\left(h, y, D_{y}\right)\left(D_{\lambda}\right)^{k} .
$$

Note that $P_{0}$ is a noncommutative symbol on $X$ and that $\left.P_{0}\right|_{h=0}=p\left(0, y, D_{y}, D_{\lambda}\right)$ is exactly the indicial operator of $Q$ [14]. Moreover, the full ellipticity of $Q$ as a $b$-operator (that is its interior ellipticity and the invertibility of the indicial family) is the same as the ellipticity of $P_{0}$ as a noncommutative symbol on $X$. Hence, we have defined a map

$$
\sigma: Q \mapsto P_{0}
$$

defined on $b$-differential operators and taking values in noncommutative symbols on $X$. It remains to extend this map to the pseudodifferential case: the idea is basically the same but things are more technical.

We will use a cover of $M \times M$ by three open subsets $R_{1}, R_{2}, R_{3}$ as shown below and a partition of unity $\omega_{1}, \omega_{2}, \omega_{3}$ subordinated to this cover. For instance, $R_{1}=\left(\left[0,1 / 2[\times L)^{2}\right.\right.$ while

$$
R_{2}=\left\{\left(x, x^{\prime}\right) \in M^{2} \mid \operatorname{dist}(x, y)<1, h(x)+h\left(x^{\prime}\right)>3 / 2\right\}
$$

and $R_{3}$ is some open neighborhood of the complement of $R_{1} \cup R_{2}$ into $M^{2}$.

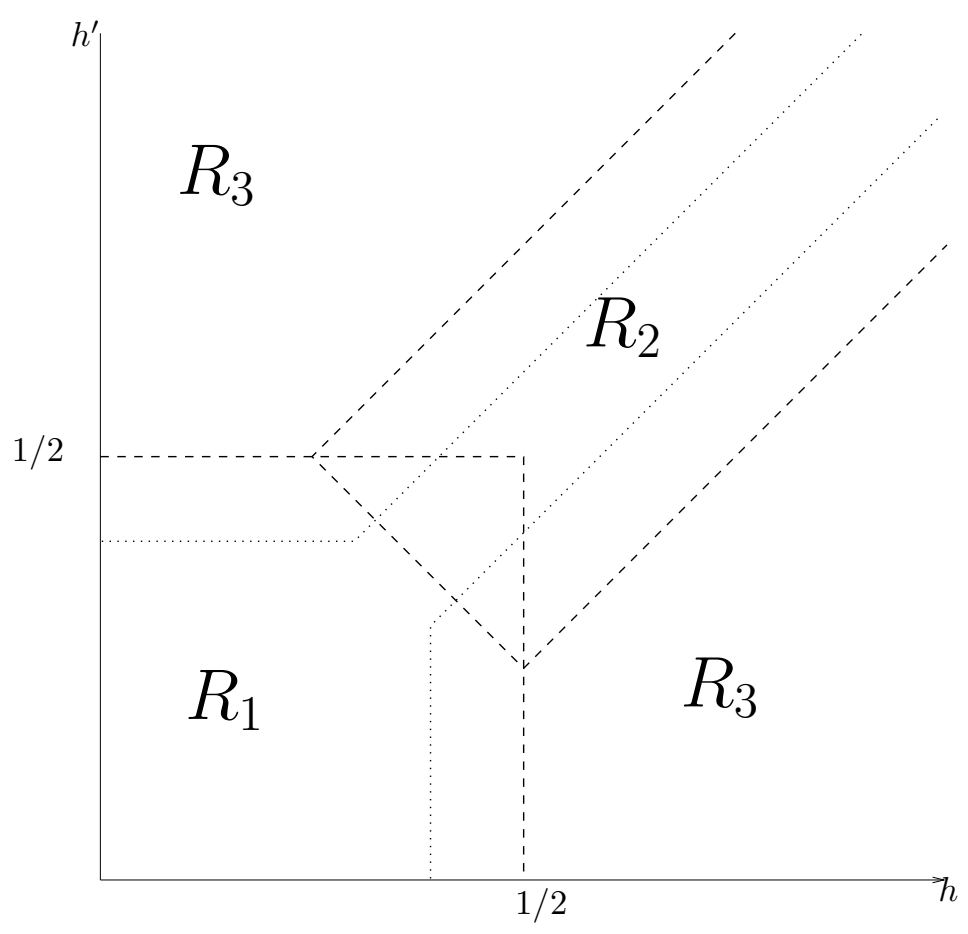

Let $Q \in \mathcal{P}_{b}^{*}(M)$ with Schwartz kernel $\kappa$. Let $Q_{i} i=1,2,3$ be the operators with Schwartz kernel $\kappa_{i}=\omega_{i} \kappa$ so that: $Q=Q_{1}+Q_{2}+Q_{3}$.

Let us focus on $Q_{1}$. Applying a Mellin transfom on $\kappa_{1}$, we get:

$$
a_{1}\left(h, \eta, y, y^{\prime}\right)=\int_{\mathbb{R}_{+}^{*}}\left(\frac{h}{h^{\prime}}\right)^{i \eta} \kappa_{1}\left(h, y, h^{\prime}, y^{\prime}\right) \frac{d h^{\prime}}{h^{\prime}}
$$

where $a_{1}$ is a smooth function of $h \in[0,1 / 2$ [ taking values in the space of pseudodifferential operators on $L$ with one parameter $\eta \in \mathbb{R}$. Using a cut-off function $\phi_{1}$ such that $\omega_{1} \phi_{1}=\omega_{1}$, one recovers the action of $Q_{1}$ on functions as follows:

$$
u \in C_{c}^{\infty}\left(X^{o}\right), \quad Q_{1} u(h, y)=\int_{\mathbb{R}_{+}^{*} \times \mathbb{R}}\left(\frac{h}{h^{\prime}}\right)^{i \eta}\left(a_{1}(h, \eta) \cdot\left(\phi_{1}\left(h, h^{\prime}\right) u\left(h^{\prime}, .\right)\right)\right)(y) \frac{d h^{\prime}}{h^{\prime}} d \eta
$$


where $\left(a_{1}(h, \eta) \cdot u\left(h^{\prime},.\right)\right)(y)$ is the action of the operator $a_{1}(h, \eta)$ on $u\left(h^{\prime},.\right) \in C^{\infty}(L)$ evaluated at $y \in L$. Since $\kappa(t, h)=1$ and $l(h)=\log (h)$ when $h \in[0,1 / 2]$, setting:

$$
u \in C_{c}^{\infty}\left(X^{o}\right), \quad P_{1, t} u(h, y)=\int_{\mathbb{R}_{+}^{*} \times \mathbb{R}}\left(\frac{h}{h^{\prime}}\right)^{i \eta / t}\left(a_{1}(h, \eta) \cdot \phi_{1}\left(h, h^{\prime}\right) u\left(h^{\prime}, .\right)\right)(y) \frac{d h^{\prime}}{t h^{\prime}} d \eta
$$

for $t>0$ and defining $P_{1,0} \in \Psi^{*}\left(\overline{T^{q} X}\right)$ by:

$$
u \in C_{c}^{\infty}(] 0,1 / 2[\times L \times \mathbb{R}), \quad P_{1,0} u(h, x, \lambda)=\int_{\mathbb{R}^{2}} e^{i\left(\lambda-\lambda^{\prime}\right) \cdot \eta}\left(a_{1}(h, \eta) \cdot u\left(h, ., \lambda^{\prime}\right)\right)(x) d \lambda^{\prime} d \eta
$$

we get a pseudodifferential operator on $\mathcal{G}^{q}$ given by $P_{1}=\left(P_{1, t}\right)_{t \in[0,1]}$ and such that $\left.P_{1}\right|_{t=1}=$ $Q_{1}$.

Since $\kappa_{2}$ is supported in $R_{2}$ which is included both in a compact subset of $X^{o} \times X^{o}$ and in the range of $E_{\mathcal{G}^{q}}=\Theta^{-1}$, we can set:

$$
\widehat{a_{2}}(x, V)=\kappa_{2}\left(E_{\mathcal{G}^{q}}(x, V, 1)\right) \text { and } a_{2}(x, \xi)=\int_{M} e^{i \Theta\left(x, x^{\prime}, 1\right) \cdot \xi} \widehat{a_{2}}\left(x, \Theta\left(x, x^{\prime}, 1\right)\right) d \lambda^{1}\left(x^{\prime}\right) .
$$

Then, choosing any function $\phi_{2}$ compactly supported in a neighborhood of $R_{2}$ and satisfying $\omega_{2} \phi_{2}=\omega_{2}$, we have for all functions $u \in C_{c}^{\infty}\left(X^{o}\right)$ :

$$
Q_{2} u(x)=\int_{M \times T_{x}^{*} M} e^{i \Theta\left(x, x^{\prime}, 1\right) \cdot \xi} a_{2}(x, \xi) \phi_{2}\left(x, x^{\prime}\right) u\left(x^{\prime}\right) d \lambda^{1}\left(x^{\prime}\right) d \xi .
$$

To extend $Q_{2}$ as we did for $Q_{1}$, we set:

$$
P_{2, t} u(x)=\int_{M \times T_{x}^{*} M} e^{i \Theta\left(x, x^{\prime}, t\right) \cdot \xi} a_{2}(x, \xi) \phi_{2}\left(x, x^{\prime}\right) u\left(x^{\prime}\right) d \lambda^{t}\left(x^{\prime}\right) d \xi .
$$

This is for $t>0$, and we define $P_{2,0} \in \Psi\left(\overline{T^{q} X}\right)$ by:

$h<1, u \in C_{c}^{\infty}(\mathbb{R} \times L),\left.P_{2,0}\right|_{h} u(\lambda, y)=\int_{\mathbb{R} \times L \times T_{(h, y)}^{*} M} e^{i \Theta\left(h, \lambda-\lambda^{\prime}, y, y^{\prime}, 0\right) \cdot \xi} a_{2}(h, y, \xi) u\left(\lambda^{\prime}, y^{\prime}\right) d \lambda^{h, 0}\left(\lambda^{\prime}, y^{\prime}\right) d \xi$

and

$$
u \in C_{c}^{\infty}\left(T X_{+}\right),\left.\quad P_{2,0}\right|_{X_{+}} u(x, X)=\int_{T_{x} M \times T_{x}^{*} M} e^{i \Theta\left(x, X-X^{\prime}, 0\right) \cdot \xi} a_{2}(x, \xi) u\left(x, X^{\prime}\right) d \lambda^{x}\left(X^{\prime}\right) d \xi .
$$

The last piece $Q_{3}$ is smoothing and its Schwartz kernel $\kappa_{3}\left(x, x^{\prime}\right)$ vanishes both on a neighborhood of the diagonal and on a neighborhood of $\partial M \times \partial M$ in $M^{2}$. This implies that $\widetilde{\kappa_{3}}$ defined by $\widetilde{\kappa_{3}}\left(x, x^{\prime}, t\right)=\kappa_{3}\left(x, x^{\prime}\right)$ if $t>0$ and $\left.\widetilde{\kappa_{3}}\right|_{t=0}=0$, belongs to $C^{\infty}\left(\mathcal{G}^{q}\right)$ and the behavior of $\kappa_{3}\left(x, x^{\prime}\right)$ near $h(x)=h\left(x^{\prime}\right)=0$, resulting from the assumption that $Q$ is in the small calculus, yields also $\kappa_{3}\left(x, x^{\prime}, t\right) \in C^{*}\left(\mathcal{G}^{q}\right)$. Thus setting for $t>0$ and $u \in C_{c}^{\infty}\left(X^{o}\right)$ :

$$
P_{3, t} u(x)=\int_{M} \kappa_{3}\left(x, x^{\prime}\right) u\left(x^{\prime}\right) d \lambda^{t}\left(x^{\prime}\right)
$$

and for $t=0: P_{3,0}=0$, we have extended $Q_{3}$ in $P_{3} \in \Psi^{-\infty}\left(\mathcal{G}^{q}\right) \cap C^{*}\left(\mathcal{G}^{q}\right)$. We get a linear map:

$$
\begin{array}{ccc}
\mathcal{P}_{b}^{*}(M) & \longrightarrow & \Psi^{*}\left(\mathcal{G}^{q}\right) \\
Q & \longmapsto & P_{Q}:=P_{1}+P_{2}+P_{3} .
\end{array}
$$

Restricting $P_{Q}$ at $t=0$ gives the desired noncommutative symbol map: 
Definition 6 Let $Q \in \mathcal{P}_{b}^{*}(M)$. With the notations above, we define the noncommutative symbol of $Q$ by :

$$
\sigma(Q)=\left.P\right|_{t=0}=P_{1,0}+P_{2,0} \in S^{*}(X) .
$$

The following facts are obvious:

Remark 3 1. $Q$ is fully elliptic as a b-operator if and only if $\sigma(Q)$ is elliptic as a noncommutative symbol on $X$.

2. If $P \in \mathcal{P}_{b}^{p}(M)$ and $Q \in \mathcal{P}_{b}^{q}(M)$ then

$$
\sigma(P Q)=\sigma(P) \sigma(Q) \text { modulo } S^{p+q-1}(X) .
$$

3. Everything above can be written in the same way for operators acting on sections of a vector bundle.

The noncommutative symbol map depends on the choices of the cover of $M^{2}$, of the partition of unity and of the exponential map of $\mathcal{G}^{q}$, but the $K$-theory class of the noncommutative symbols of fully elliptic $b$-operators does not depend on these choices, as we will see in the next paragraph.

Conversely, one can define a quantification map op ${ }_{b}$ which is a quasi inverse of $\sigma$. We describe it now. Let us choose $\omega \in C_{c}^{\infty}([0,1 / 2[)$ such that $\omega(h)=1$ near $h=0$. Let $a$ be a noncommutative symbol on $X$ and write

$$
a=\omega a+(1-\omega) a=a_{1}+a_{2} .
$$

We extend $a_{1}$ as a $\mathcal{G}^{q}$-pseudodifferential operator $\widetilde{a_{1}}$ by reverting the process used in (4.54.6). Let $f_{2}$ be a symbol of $a_{2}$ viewed as a pseudodifferential operator on $T^{q} X$. That means that $f_{2}$ is an ordinary symbol on $A^{*}\left(T^{q} X\right)=T^{*} X^{o}$ such that:

$$
a_{2}=\mathrm{op}_{T^{q} X}\left(f_{2}\right) \text { modulo } S_{0}^{-\infty}(X, E)
$$

where $\mathrm{op}_{T^{q} X}$ is given by:

$u \in C_{c}^{\infty}\left(T^{q} X\right), \mathrm{op}_{T^{q} X}\left(f_{2}\right)(u)(\gamma)=\int_{\left(T^{q} X\right)_{s(\gamma)} \times T_{r(\gamma)}^{*} X^{o}} e_{i<\Theta^{q}\left(\gamma^{\prime} \gamma^{-1}\right), \xi>} f_{2}(r(\gamma), \xi) \phi\left(\gamma^{\prime} \gamma^{-1}\right) u\left(\gamma^{\prime}\right) d \lambda^{s(\gamma)} d \xi$.

Here $\Theta^{q}=\left(E_{T^{q} X}\right)^{-1}$ is the inverse of the exponential map of $T^{q} X$ given by restriction of $E_{\mathcal{H}}$, and $\phi$ is a cut-off function equal to 1 on units and supported in the range of $\Theta^{q}$. We can use the formulae (4.7/4.8, 4.9) to build from $f_{2}$ a $\mathcal{G}^{q}$-pseudodifferential operator $\widetilde{a_{2}}$ with the property :

$$
\left.\widetilde{a_{2}}\right|_{t=0}=a_{2} \text { modulo } S_{0}^{-\infty}(X) .
$$

Thus we get an approximate lifting of noncommutative symbols:

$$
\widetilde{a}:=\widetilde{a_{1}}+\widetilde{a_{2}} \in \Psi_{b}^{*}\left(\mathcal{G}_{q}\right)
$$

satisfying:

$$
\begin{gathered}
\left.\widetilde{a}\right|_{t=0}=a \text { modulo } S_{0}^{-\infty}(X), \\
\operatorname{op}_{b}(a):=\left.\widetilde{a}\right|_{t=1} \in \mathcal{P}_{b}^{*}(M) .
\end{gathered}
$$

By construction:

$$
\sigma\left(\operatorname{op}_{b}(a)\right)=a \text { modulo } S_{0}^{-\infty}(X)
$$

In the same way, if $P \in \mathcal{P}_{b}^{*}(M)$ then $\mathrm{op}_{b}(\sigma(P))-P$ is a smoothing operator with vanishing indicial operator. 


\subsection{The Poincaré duality as a noncommutative symbol map}

All ingredients are now at hands to finish. Observe that to each fully elliptic $b$-operator $P$ : $C^{\infty}(M, E) \rightarrow C^{\infty}(M, F)$ acting on sections of complex vectors bundles $E, F$, corresponds a $K$-homology class $[P]=\left[\left(L^{2}\left(M ; E \oplus F ; d \lambda^{1}\right), \rho_{1}, \mathbf{P}\right)\right]$ on $X$ where:

- $L^{2}\left(M ; E \oplus F ; d \lambda^{1}\right)$ is the $\mathbb{Z}_{2}$-graded Hilbert space modeled on the measure $d \lambda^{1}$ and on product type hermitian structures on $E$ and $F$,

- $\rho_{1}$ is the action of $C(X)$ onto $L^{2}\left(M ; E \oplus F ; d \lambda^{1}\right)$ in the natural way through the quotient map $M \rightarrow X=M / \overline{X_{-}}$,

- $\mathbf{P}=\left(\begin{array}{cc}0 & Q \\ P & 0\end{array}\right)$ where $Q$ is a full parametrix of $P$.

Theorem 2 With the previous notations and those of the definition 4 , the isomorphism $\Sigma^{q}: K_{0}(X) \rightarrow K^{0}\left(T^{q} X\right)$ defined in (2.2) is given by:

$$
[P] \longmapsto[\sigma(P)]
$$

\section{Remark 4}

- Recall that from theorem [1, we know that every $K$-theory class $[a] \in K^{0}\left(T^{q} X\right)$ has a representant a among elliptic noncommutative symbols on $X$. From (4.12), we know that $a$ is in the same $K$-theory class than the noncommutative symbol $\sigma(P)$ of a fully elliptic b-operator P. Eventually, since $\Sigma^{q}$ is an isomorphism, we get that each $K$-homology class of $X$ is represented by a fully elliptic b-operator.

- Using the deformation of $T^{q} X$ into $T^{c} X$, leading to the $K K$-equivalence $T^{q} X \sim T^{c} X$, one could also get a concrete interpretation for $\Sigma^{c}$. However, the adequate adaptation of the notion of noncommutative symbols is more difficult to relate directly to what is done in boundary values problems or former studies about pseudodifferential calculus for groupoids.

Proof : Let $P: C^{\infty}\left(M, E_{0}\right) \rightarrow C^{\infty}\left(M, E_{1}\right)$ be a fully elliptic 0-order b-operator. Let $E=E_{0} \oplus E_{1}$ and $a=\sigma(P) \in S^{0}(X, E)$. We need to prove that $\Sigma^{q}[P]=[a]$ or equivalently that $[P]=[a] \underset{T^{q} X}{\otimes} D^{q}$ (cf. section 2). Recall that:

$$
[a] \underset{T^{q} X}{\otimes} D^{q}=\mathbf{s}_{X}([a]) \otimes \Phi^{q} \otimes \partial^{q} .
$$

Firstly, $\mathbf{s}_{X}([a]) \otimes \Phi^{q} \in K K\left(X, T^{q} X\right)$ is represented by:

$$
\left(C^{*}\left(T^{q} X, E\right), \rho, \mathbf{a}\right)
$$

where $\rho: C(X) \rightarrow \mathcal{L}\left(C^{*}\left(T^{q} X, E\right)\right)$ is given by $\rho(f)(\xi)(\gamma)=\xi(\gamma) f\left(\pi^{q}(\gamma)\right)$.

The next step is to find

$$
[\widetilde{\mathcal{E}}, \widetilde{\rho}, \widetilde{\mathbf{a}}] \in K K\left(X, \mathcal{G}^{q}\right)
$$

such that

$$
\left(e_{0}^{q}\right)_{*}[\widetilde{\mathcal{E}}, \widetilde{\rho}, \widetilde{\mathbf{a}}]=\left[C^{*}\left(T^{q} X, E\right), \rho, \mathbf{a}\right] .
$$

The desired lifting is made as follows. Let us note again $E$ the pull back of the original bundle $E$ to $X^{o} \times[0,1]$ with the range map of $\mathcal{G}^{q}$. Let $\widetilde{\pi^{q}}$ be the composite map of the range map $\mathcal{G}^{q} \rightarrow X^{o} \times[0,1]$ with the projection maps $X^{o} \times[0,1] \rightarrow X^{o}$ and $X^{o} \rightarrow X=X^{o} / \overline{X_{-}}$.

We set $\widetilde{\mathcal{E}}=C^{*}\left(\mathcal{G}^{q}, E\right)$, we define $\widetilde{\rho}$ by $\widetilde{\rho}(f)(\xi)(\gamma)=f\left(\widetilde{\pi^{q}}(\gamma)\right) \xi(\gamma)$, and $\widetilde{\mathbf{a}}$ is defined from a using (4.10). By construction $\widetilde{\mathbf{a}} \in \Psi_{b}^{0}\left(\mathcal{G}^{q}, E\right) \subset \mathcal{L}\left(C^{*}\left(\mathcal{G}^{q}, E\right)\right)$ and $\widetilde{\mathbf{a}}^{2}=1$ modulo 
$\Psi_{b, 0}^{-1}\left(\mathcal{G}^{q}, E\right) \subset \mathcal{K}\left(C^{*}\left(\mathcal{G}^{q}, E\right)\right)$. It follows that the triple $(\widetilde{\mathcal{E}}, \widetilde{\rho}, \widetilde{\mathbf{a}})$ defined above satisfies (4.16) and (4.17). Evaluating this element at $t=1$ gives:

$$
\left(e_{1}^{q}\right)_{*}[\widetilde{\mathcal{E}}, \widetilde{\rho}, \widetilde{a}]=\left[\left.\widetilde{\mathcal{E}}\right|_{t=1}, \widetilde{\rho}_{t=1}, \mathrm{op}_{b}(a)\right] \in K K\left(X, \mathcal{C}_{X^{o}}\right)
$$

and applying the Morita equivalence $C^{*}\left(\mathcal{C}_{X^{\circ}}\right) \stackrel{\nu}{\sim} \mathbb{C}$ produces the final result:

$[a] \underset{T^{q} X}{\otimes} D^{q}=\left[\left.\widetilde{\mathcal{E}}\right|_{t=1}, \widetilde{\rho}_{t=1}, \mathrm{op}_{b}(a)\right] \otimes \nu=\left[L^{2}\left(M ; E ; d \lambda^{1}\right), \rho_{1}, P\right]=[P] \in K K(X, \cdot)=K_{0}(X)$

\subsection{Index map}

Since $X$ is a compact Hausdorff space the map $p$ sending $X$ to a point gives rise to a morphism:

$$
p_{*}: K_{0}(X) \rightarrow K_{0}(\cdot)=\mathbb{Z}
$$

called, for obvious reasons, the index map. We can capture $p_{*}$ with the pre-Dirac element and Poincaré duality:

Proposition 9 Let us denote by $\operatorname{Ind}^{q}$ the map:

$$
\operatorname{Ind}^{q}:[a] \in K\left(T^{q} X\right) \mapsto[a] \otimes \partial^{q} \in \mathbb{Z},
$$

then the following holds:

$$
\forall[a] \in K\left(T^{q} X\right), \quad p_{*}\left(\left(\Sigma^{q}\right)^{-1}[a]\right)=\operatorname{Ind} d^{q}[a] .
$$

In other words, the index of a fully elliptic 0-order b-operator $P: C^{\infty}(M, E) \rightarrow C^{\infty}(M, F)$ viewed as a Fredholm operator between $L^{2}\left(M, E, d \lambda^{1}\right)$ and $L^{2}\left(M, F, d \lambda^{1}\right)$ is equal to Ind $d^{q}(\sigma(P))$.

Proof : The homomorphism $\mathbb{C} \rightarrow C(X)$ corresponding to $p: X \rightarrow \cdot$ is denoted by $\widetilde{p}$. We have

$$
p_{*}\left(\left(\Sigma^{q}\right)^{-1}[a]\right)=[\widetilde{p}] \otimes\left([a] \underset{T^{q} X}{\otimes}\left(\left[\Phi^{q}\right] \otimes \partial^{q}\right)\right)=[\widetilde{p}] \otimes \mathbf{s}_{X}[a] \otimes\left[\Phi^{q}\right] \otimes \partial^{q} .
$$

Observe that

$$
[\widetilde{p}] \otimes \mathbf{s}_{X}[a]=[\widetilde{p}] \underset{\mathbb{C}}{\otimes}[a]
$$

so by the commutativity of the Kasparov product over $\mathbb{C}$ :

$$
[\widetilde{p}] \otimes \mathbf{s}_{X}[a]=\otimes[a] \underset{\mathbb{C}}{\otimes}[\widetilde{p}]=a \otimes \mathbf{s}_{T^{q} X}[\widetilde{p}] .
$$

But $\mathbf{s}_{T^{q} X}[\widetilde{p}] \otimes\left[\Phi^{q}\right]$ is equal to the class of the identity homomorphism of $C^{*}\left(T^{q} X\right)$, hence:

$$
\begin{aligned}
p_{*}\left(\left(\Sigma^{q}\right)^{-1}[a]\right) & =\left([\widetilde{p}] \otimes \mathbf{s}_{X}[a]\right) \otimes\left[\Phi^{q}\right] \otimes \partial^{q} \\
& =\left(a \otimes \mathbf{s}_{T^{q} X}[\widetilde{p}]\right) \otimes\left[\Phi^{q}\right] \otimes \partial^{q} \\
& =a \otimes\left(\mathbf{s}_{T^{q} X}[\widetilde{p}] \otimes\left[\Phi^{q}\right]\right) \otimes \partial^{q} \\
& =a \otimes \partial^{q}
\end{aligned}
$$




\section{References}

[1] C. Anantharaman-Delaroche and J. Renault. Amenable groupoids, volume 282 of Contemp. Math. Amer. Math. Soc., Providence, RI, 2001.

[2] S. Baaj and P. Julg. Théorie bivariante de Kasparov et opérateurs non bornés dans les $C^{*}$-modules hilbertiens. C.R. Acad Sc. Paris Série I, 296:875, 878.

[3] B. Blackadar. K-theory for operator algebras, volume 5 of Mathematical Sciences Research Institute Publications. Cambridge University Press, Cambridge, second edition, 1998.

[4] J.P. Brasselet, G. Hector, and M. Saralegi. Thorme de de Rham pour les varits stratifies. Ann. Global Analy. Geom., 9(3):211-243, 1991.

[5] A. Connes. Noncommutative Geometry. Academic Press, 1994.

[6] A. Connes and G. Skandalis. The longitudinal index theorem for foliations. Publ. R.I.M.S. Kyoto Univ., 20:1139-1183, 1984.

[7] C. Debord and J.M. Lescure. $K$-duality for pseudomanifolds with isolated singularities. J. Funct. Anal., 219(1):109-133, 2005.

[8] C. Debord, J.M. Lescure, and V. Nistor. Groupoids and an index theorem for conical pseudo-manifolds. Preprint, http://arxiv.org/abs/math/0609438v1, 2006.

[9] G. Elliott, T. Natsume, and R. Nest. The Atiyah-Singer index theorem as passage to the classical limit in quantum mechanics. Comm. Math. Phys., 182(3):505-533, 1996.

[10] E. Getzler. Pseudodifferential operators on supermanifolds and the Atiyah-Singer index theorem. Commun. Math. Phys., 92:163-178, 1983.

[11] G.G. Kasparov. The operator K-functor and extensions of $C^{*}$-algebras. Izv. Akad. Nauk SSSR, Ser. Math., 44:571-636, 1980.

[12] G.G. Kasparov. Equivariant KK-theory and the Novikov conjecture. Invent. math., 91:147-201, 1988.

[13] R. Lauter, B. Monthubert, and V. Nistor. Spectral invariance for certain algebras of pseudodifferential operators. J. Inst. Math. Jussieu, 4(3):405-442, 2005.

[14] R.B. Melrose. The Atiyah-Patodi-Singer index theorem, volume 4 of Research Notes in Mathematics. A. K. Peters, Massachusetts, 1993.

[15] R.B. Melrose. The eta invariant and families of pseudodifferential operators. Math. Res. Lett., 2(5):541-561, 1995.

[16] B. Monthubert. Groupoids of manifolds with corners and index theory. In Groupoids in analysis, geometry, and physics (Boulder, CO, 1999), pages 147-157. Amer. Math. Soc., Providence, RI, 2001.

[17] B. Monthubert. Groupoids and pseudodifferential calculus on manifolds with corners. J. Funct. Anal., 199(1):243-286, 2003.

[18] B. Monthubert and F. Pierrot. Indice analytique et groupoïde de Lie. C.R.A.S Série 1, 325:193-198, 1997.

[19] V. E. Nazaikinskii, A. Yu. Savin, and B. Yu. Sternin. On the homotopy classification of elliptic operators on stratified manifolds. Preprint, math.KT/0608332, 2006. 
[20] V. E. Nazaikinskii, A. Yu. Savin, and B. Yu. Sternin. Pseudodifferential operators on stratified manifolds. Preprint, math.AP/0512025, 2006.

[21] V. Nistor, A. Weinstein, and P. Xu. Pseudodifferential operators on differential groupoids. Pacific J. of Math., 181(1):117-152, 1999.

[22] A. Savin. Elliptic operators on manifolds with singularities and $K$-homology. $K$ Theory, 34(1):71-98, 2005.

[23] B.W. Schulze. Pseudodifferential boundary value problems, conical singularities, and asymptotics, volume 4 of mathematical topics. Akademie Verlag, 1994.

[24] B.W. Schulze. Boundary value problems and singular pseudodifferential operators. Wiley-Intersciences, 1998.

[25] M.A. Shubin. Pseudodifferential operators and spectral theory. Springer Verlag, 1980.

[26] G. Skandalis. Kasparov's bivariant K-theory and applications. Expositiones mathematicae, 9:193-250, 1991.

[27] S. Vassout. Unbounded pseudodifferential calculus on Lie groupoids. to appear in Journal of Functional Analysis.

[28] S. Vassout. Feuilletages et Résidu non Commutatif Longitudinal. PhD thesis, Université Paris VI, 2001.

[29] N. E. Wegge-Olsen. K-theory and $C^{*}$-algebras. Oxford Science Publications. The Clarendon Press Oxford University Press, New York, 1993. A friendly approach.

[30] H. Widom. A complete symbolic calculus for pseudodifferential operators. Bull. Sci. Math. (2), 104(1):19-63, 1980. 